\title{
Good Practices for Estimating Area and Assessing Accuracy of Land Change
}

Pontus Olofsson ${ }^{\mathrm{a}, *}$, Giles M. Foody ${ }^{\mathrm{b}}$, Martin Herold ${ }^{\mathrm{c}}$, Stephen V. Stehman ${ }^{\mathrm{d}}$, Curtis E.

Woodcock $^{\mathrm{a}}$ and Michael A. Wulder ${ }^{\mathrm{e}}$

${ }^{a}$ Department of Earth and Environment, Boston University, 685 Commonwealth Avenue,

Boston, MA 02215, USA

${ }^{b}$ School of Geography, University of Nottingham, University Park, Nottingham NG7 2RD, UK

${ }^{c}$ Laboratory of Geo-Information Science and Remote Sensing, Wageningen University,

Droevendaalsesteeg 3, 6708 Wageningen, The Netherlands

${ }^{d}$ Department of Forest and Natural Resources Management, State University of New York, 1

Forestry Drive, Syracuse, NY 13210, USA

${ }^{e}$ Canadian Forest Service (Pacific Forestry Centre), Natural Resources Canada, Victoria, BC, 12 V8Z 1M5, Canada

Key words: accuracy assessment, sampling design, response design, area estimation, land change, remote sensing

*Corresponding author

Email olofsson@bu.edu

URL people.bu.edu/olofsson

Phone +1-617-353-9734

Fax +1-617-353-8399 


\section{Abstract}

2 The remote sensing science and applications communities have developed increasingly reliable,

3 consistent, and robust approaches for capturing land dynamics to meet a range of information

4 needs. Statistically robust and transparent approaches for assessing accuracy and estimating area

5 of change are critical to ensure the integrity of land change information. We provide

6 practitioners with a set of "good practice" recommendations for designing and implementing an

7 accuracy assessment of a change map and estimating area based on the reference sample data.

8 The good practice recommendations address the three major components: of the process

9 including the-sampling design, response design and analysis. The primary good practice

10 recommendations for assessing accuracy and estimating area are: (i) implement a probability

11 sampling design that is chosen to achieve the priority objectives of accuracy and area estimation

12 while also satisfying practical constraints such as cost and available sources of reference data;

13 (ii) implement a response design protocol that is based on reference data sources that provide

14 sufficient spatial and temporal representation to accurately label each unit in the sample (i.e., the

15 "reference classification" will be considerably more accurate than the map classification being

16 evaluated); (iii) implement an analysis that is consistent with the sampling design and response

17 design protocols; (iv) summarize the accuracy assessment by reporting the estimated error matrix

18 in terms of proportion of area and estimates of overall accuracy, user's accuracy (or commission

19 error), and producer's accuracy (or omission error); (v) estimate area of classes (e.g., types of

20 change such as wetland loss or types of nochangepersistence such as stable forest) based on the

21 reference classification of the sample units; (vi) quantify uncertainty by reporting confidence

22 intervals for accuracy and area parameters; (vii) evaluate variability and potential error in the 
23 reference classification; and (viii) document deviations from good practice that may substantially

24 affect the results. An example application is provided to illustrate the recommended process. 


\section{1. Introduction}

26 Land change maps quantify a wide range of processes including wildfire (Schroeder et al., 2011),

27 forest harvest (Olofsson et al., 2011), forest disturbance (Huang et al., 2010), land use pressure

28 (Drummond and Loveland, 2010) and urban expansion (Jeon et al., 2013). Map users and

29 producers are acutely interested in communicating and understanding the quality of these maps.

30 Accordingly, guidance on how to assess accuracy of these maps in a consistent and transparent

31 manner is a necessity. The use of remote sensing products depicting change for scientific,

32 management, or policy support activities-all require quantitative accuracy statements to buttress

33 the confidence in the information generated and in any subsequent reporting or inferences made.

34 Area estimation, whether of change in land cover/use or of status of land cover/use at a single

35 date, is a natural value-added use of land change maps in many local, national and global land

36 accounting applications. For example, the amount of land area allocated for a specific use is a

37 key country reporting requirement to the United Nations (UN) Food and Agriculture

38 Organization (FAO) statistics and the global forest resources assessment (FAO, 2010) and as

39 well as for countries reporting under the Kyoto protocol and the evolving activities for the UN

40 Collaborative Programme on Reducing Emissions from Deforestation and Forest Degradation -

41 UN-REDD (UN-REDD, 2008; Grassi et al., 2008). Estimates of forest extent or deforestation are

42 often derived via remote sensing (cf. Achard et al., 2002; DeFries et al., 2002; Hansen et al.,

43 2010), and area estimation also plays a prominent role in ongoing efforts to establish

44 scientifically valid protocols for forest change monitoring in the context of specific accounting

45 applications to policy approaches for reducing greenhouse gas emissions from forests (DeFries et

46 al., 2007; GOFC-GOLD, 2011). 
Area estimation also plays a prominent role in ongoing efforts to establish scientifically valid

48 protocols for forest change monitoring in the context of specific accounting applications to

49 policy approaches for reducing greenhouse gas emissions from forests (DeFries et al., 2007;

50 GOFC-GOLD, 2011). One approach to quantifying greenhouse gas emissions from forests, an

51 important component of carbon accounting, is based on estimating the area of forest change and

52 then applying emissions factors associated with these changes to translate the area changes into

53 emissions (Herold and Skutsch, 2011).-Thus, understanding the uncertainty in area change

54 estimates is one key factor determining the accuracy of the overallemission and for assessing the

55 performance and impact of climate change mitigation activities to reduce these emissions

56 (GOFC GOLD, 2011; Herold et al., 2011). Furthermore, the efforts of the UN REDD-clearly call

57 for area estimates of deforestation and degradation with known uncertainty (UN REDD, 2008).

58 The reporting obligations of national governments also benefit from a capacity to quantitatively

59 report on accuracy of products and to build confidence in the reported outcomes (Wulder et al.,

60 2007). Forest certification programs, aimed at ensuring sustainable forest management practices,

61 also require scientifically accepted means for monitoring land based changes in a transparent and

62 quantifiable manner.

63 A key strength of remote sensing is that it enables spatially exhaustive, wall-to-wall

64 coverage; of the area of interest. Buthowever, as might be expected with any mapping process,

65 the results are rarely perfect. Placing spatially and categorically continuous conditions into

66 discrete classes will-may result in confusion at the categorical transitions. Error can also result

67 from the change mapping process, the data used, and analyst biases (Foody, 2010). Change

68 detection and mapping approaches using remotely sensed data are increasingly robust, with

69 improvements aimed at the mitigation of these sources of error. However, any map made from 
70 remotely sensed data can be assumed to contain some error, with the areas calculated from the

71 map (e.g., pixel counting) also potentially subject to bias. An accuracy assessment identifies the

|72 errors of the classification ${ }_{2}$ and the sample data can be used for estimating both accuracy and

73 area along with the uncertainty of these estimates. While the notion of accuracy assessment is

74 well-established within the remote sensing community (Foody, 2002; Strahler et al., 2006),

75 studies of land change routinely fail to assess the accuracy of the final change maps and few

76 published studies of land change make full use of the information obtained from accuracy

77 assessments (Olofsson et al., 2013).

\section{$78 \quad 1.1$ Good Practice Recommendations}

79 In this article, we synthesise the current status of key steps and methods that are needed to 80 complete an accuracy assessment of a land change map and to estimate area of land change. The

81 This article addresses the fundamental protocols required to produce scientifically rigorous and

82 transparent estimates of accuracy and area. The set of good practice recommendations provides

83 guidelines to assist both scientists and practitioners in the design and implementation of accuracy

84 assessment and area estimation methods applied to land change assessments using remote

85 sensing. The accuracy and area estimation objectives are linked via a map of change. A change

86 map provides a spatially explicit depiction of change and this spatial information can be readily

87 aggregated to calculate the total mapped area or the proportion of mapped area of change for the

88 region of interest (ROI). Accuracy assessment addresses questions related to how well locations

89 of mapped change correspond to actual areas of change. A fundamental premise of the

90 recommended good practices methodology is that the change map will be subject to an accuracy

91 assessment based on a sample of higher quality change information (i.e., the reference

92 classification). The higher quality reference classification is compared to the map classification 
93 on a location-specific basis to quantify accuracy of the change map and to estimate area.

94 Although it is possible to estimate area of change without producing a change map (Achard et

95 al., 2002; FAO, 2010; Hansen et al., 2010), we will assume that a map of change exists (although

96 there will not necessarily be a map for each date). The focus for this document is change between

97 two dates.

98 At the outset $b$ Before any detailed planning of the response and sampling designs is

99 undertaken, a basic visual assessment should be conducted to identify obvious errors and

100 concerns in the remotely sensed product. This assessment provides an evaluation of the map's

101 suitability for the intended application and should detect if a map is so unsuitable for use that

102 there is no value in proceeding to a more detailed assessment. The visual assessment should also

103 highlight errors that are easy to remove enabling the map to be refined prior to initiating a

104 detailed assessment or confirm that no obvious concerns exist and the map is ready for further

105 rigorous evaluation.

106 We separate the accuracy assessment methodology into three major components, the

107 response design, sampling design, and analysis (Stehman and Czaplewski, 1998). The response

108 design encompasses all aspects of the protocol that lead to determining whether the map and

109 reference classifications are in agreement. Because it is often impractical to apply the response

110 design to the entire ROI, a subset of the area is sampled. The sampling design is the protocol for

111 selecting that subset of the ROI. The analysis includes protocols for defining how to quantify

112 accuracy along with the formulas and inference framework for estimating accuracy and area and

113 quantifying uncertainty of these estimates. A separate section of this guidance document is

114 devoted to each of these three major components of accuracy assessment methodology. These

115 sections are followed by an example of the recommended workflow. 


\subsection{Context of Good Practice Recommendations}

117 The good practice recommendations are intended to represent a synthesis of the current science

118 of accuracy assessment and area estimation. We fully anticipate that improved methods will be

119 developed over time. As the designation of "best practice" implies a singular approach, we prefer

120 the use of "good practice" to indicate that "best" is relative and will vary, with one hard-coded

121 approach not always appropriate. In communicating good practices, desirable features and

122 selection criteria can be followed to ensure that the protocol applied satisfies - as thoroughly as

123 possible - the accuracy and area estimation recommendations. The good practices

124 recommendations do not preclude the existence of other acceptable practices, but instead

125 represent protocols that, if implemented correctly, would ensure scientific credibility of the

126 results. Furthermore, the recommendations presented herein allow flexibility to choose specific

127 details of the different components of the methodology. For example, while the general

128 recommendation for the sampling design is to implement a probability sampling protocol, there

129 are numerous sampling designs that meet this criterion (Stehman, 2009). Similarly, the response

130 design protocol allows flexibility to use a variety of different sources for determining the

131 reference classification and multiple options exist for defining agreement between the map and

132 reference classifications. The good practices recommendations represent an ideal to strive for,

133 but it is likely that most projects will not satisfy every recommendation. Documenting and

134 justifying deviations from good practices are expected features of many accuracy assessment and

135 area estimation studies. For the most part, the good practice recommendations consist of methods

136 for which there is considerable experience of practical use in the remote sensing community.

137 These good practice recommendations for area estimation and accuracy assessment of land

138 change build on earlier guidelines for single-date land-cover maps described by Strahler et al. 
139 (2006). Strahler et al. (2006) presented general guiding principles of good practices with less 140 emphasis on details of methodology. In the intervening years since Strahler et al. (2006),

141 additional theory and practical application related to accuracy assessment and area estimation

142 have been accumulated, and this current document avails upon these developments to delve more

143 deeply into methodological details. We do not attempt to provide an exhaustive description of

144 methods given the range of issues and the highly application-specific nature of the topic. Instead,

145 our purpose is to focus upon the main issues needed to establish a common basis of good

146 practice methodology that will be generally applicable and result in transparent methods and

147 rigorous estimates of accuracy and area. A list of recommendations for all components of the

148 process (sampling design, response design, and analysis) is presented in the Summary (Section

149 6).

150 Estimating area and accuracy of change maps introduces additional methodological

151 challenges that were not within the scope addressed by Strahler et al. (2006). In particular, the

152 area estimation objective was not addressed at all by Strahler et al. (2006). Accuracy assessment

153 of change highlights many unique challenges, including the dynamic nature of the reference data,

154 and aspects of the change features including type, severity, persistence, and area, as examples.

155 Another challenge is that change is usually a rare feature over a given landscape. The accuracy

156 of a map and the area estimates derived with its aid are a function of the land_-cover mosaic

157 under study, the underlying imagery and the methods applied. Accuracy and area estimates for

158 the same region will, for example, vary if using a per-pixel or object-based classification or if the

159 spatial resolution of the imagery is altered-and different methods vary in value for a given

160 application (cf. Duro et al., 2012; Baker et al., 2013; Johnson, 2013). 
161 The Our recommendations also focus on methods for providing robust estimates of land 162 (area) change and its uncertainties. A primary use of such estimates is in analysis and accounting

163 frameworks such as national inventories. In evolving frameworks compensating for successful

164 climate change mitigation actions in the forest sector (such as REDD+, DeFries et al., 2007), the

165 consideration of uncertainties are likely linked with financial incentives and are subject to

166 critical international political negotiations on reporting and verification (Sanz-Sanchez et al.,

167 2013). Understanding and management of uncertainties in area change is essential, in particularly

168 since because data and capacity gaps in forest monitoring are large in many developing countries

169 (Romijn et al., 2012). Accuracy assessments should also focus on identifying and addressing

170 error sources, and prioritize on capacity development needs to provide continuous improvements

171 and reduce uncertainties in the estimates over time. This also includes assessing the value of data

172 streams from evolving monitoring technologies (de Sy et al., 2012; Pratihast et al., 2013) where

173 the ultimate impact on lower uncertainties need to be proven in operational contexts. Thus, the

174 methods of good practice presented here are generic for providing robust estimates ${ }_{2}$ and having

175 agreed-upon tools to do so will provide the saliency and legitimacy for using them in quantifying

176 improvements in monitoring systems, and for dealing with uncertainties in financial

177 compensation schemes (e.g., for climate change mitigation actions).

$178 \quad$ This article synthesizes key steps and methods needed to complete an accuracy assessment of

179 a change map and to estimate area and accuracy of the map classes. It addresses the protocols

180 required to produce scientifically rigorous and transparent estimates of accuracy and area. 


\section{2. Sampling Design}

182 The sampling design is the protocol for selecting the subset of spatial units (e.g., pixels or

183 polygons) that will form the basis of the accuracy assessment. Choosing a sampling design

184 requires taking inte- consideration of the specific objectives of the accuracy assessment and a

185 prioritized list of desirable design criteria. The most critical recommendation is that the sampling

186 design should be a probability sampling design. An essential element of probability sampling is

187 that randomization is incorporated in the sample selection protocol. Probability sampling is

188 defined in terms of inclusion probabilities, where an inclusion probability relates the likelihood

189 of a given unit being included in the sample (Stehman, 2000). The two conditions defining a

190 probability sample are that the inclusion probability must be known for each unit selected in the

191 sample and the inclusion probability must be greater than zero for all units in the ROI (Stehman,

192 2001).

193 A variety of probability sampling designs are applicable to accuracy assessment and area

194 estimation ${ }_{2}$ with the most commonly used designs,- being simple random, stratified random, and

195 systematic (Stehman, 2009). Non-probability sampling protocols include purposely selecting

196 sample units (e.g., choosing units that are convenient to access mnits), restricting the sample to

197 homogeneous areas, and implementing a complex or ad hoc selection protocol for which it is not

198 possible to derive the inclusion probabilities. The condition that the inclusion probabilities must

199 be known for the units selected in the sample must be adhered to. These inclusion probabilities

200 are the basis of the estimates of accuracy and area, so if they are not known, the probabilistic

201 basis for design-based inference (see Section 4.2) is forfeited. It is difficult to envision a

202 circumstance in which a deviation from this condition of probability sampling (i.e., known

203 inclusion probabilities) would be acceptable in rigorous scientific research. 
In practice, it is not always possible to adhere perfectly to a probability sampling protocol

205 (Stehman, 2001). For example, if the response design specifies field visits to sample locations, it

206 may be too dangerous or too expensive to access some of the sample units. Conversely,

207 persistent cloud coverage or lack of useable imagery for portions of the ROI may prevent

208 obtaining the reference classification for some sample units. The reference data are often derived

209 from another set of imagery and the spatial and temporal coverage of reference data might be

210 different from the coverage of the imagery used to create the map. If the reference classification

211 for a sample unit cannot be obtained, the inclusion probability is zero for that unit. All deviations

212 from the probability sampling protocol should be documented and quantified to the greatest

213 extent possible. For example, the proportion of the selected sample units for which cloud cover

214 prevented assessment of the unit should be reported, or the proportion of area of the ROI for

215 which the reference imagery is not available should be documented. Whereas probability

216 sampling ensures representation of the population via the rigorous probabilistic basis of inference

217 established, when a large proportion of the ROI is not available to be sampled, the question of

218 how well the sample represents the population must be addressed by subjective judgment.

\section{2.1. Choosing the Sampling Design}

220 The major decisions in choosing a sampling design relate to trade-offs among different designs

221 in terms of advantages to meet specified accuracy objectives and priority desirable design

222 criteria. The objectives commonly specified are to estimate overall accuracy, user's accuracy (or 223 commission error), producer's accuracy (or omission error), and area of each class (e.g., area of

224 each type of land change). Estimates for subregions of the ROI are also often of interest (cf.

225 Scepan, 1999). Desirable sampling design criteria include: probability sampling design $; 2$, easey

226 and practicality of $t$ implementation $;-2$ cost effectiveness $;$, representative spatially well 
227 distributioned across

228 estimates, that have small standard errors; easey to-of accommodatinge a change in sample size

229 at any step in the implementation of the design $\overline{-}_{2}$ and availability of an approximately unbiased

230 estimator of variance. Determining whether eertain-any or all of these desirable design criteria

231 have been satisfied by the chosen sampling design may be subjective. For example, determining

232 what constitutes a small standard error will depend on the application and may vary for different

233 estimates within the same project. There are also precedents for defining an accuracy target and

234 desired error bounds as a means for determination of sample size using standard statistical theory

235 (Wulder et al., 2006a) (see also Section 5.1.1).

236 Stehman and Foody (2009) provide an overview and comparison of the basic sampling

237 designs typically applied to accuracy assessment. Stehman (2009) provides a more expansive

238 review of sampling design options and discusses how these designs fulfill different objectives

239 and desirable design criteria. A variety of sampling designs will satisfy good practice guidelines

240 so the key is to choose a design well suited for a given application. Three key decisions that

241 strongly influence the choice of sampling design are whether to use strata, whether to use

242 clusters, and whether to implement a systematic or simple random selection protocol (Stehman,

243 2009). Each of these decisions will be discussed in the following subsections.

244 2.1.1. Strata

245 There is $\Theta$ ften often there is a desire to partition the ROI into discrete, mutually exclusive 246 subsets or strata (e.g., a global map could be stratified geographically by continents).

247 Stratification is a partitioning of the ROI in which each assessment unit is assigned to a single

248 stratum. The two most common attributes used to construct strata are the classes determined

249 from the map and geographic subregions within the ROI. Stratification is implemented for two 
250 primary purposes. The first purpose is when the strata are of interest for reporting results (e.g.,

251 accuracy and area are reported by land--cover class or by geographic subregion). The second use

252 of stratification is to improve the precision of the accuracy and area estimates. For example,

253 when strata are created for the objective of reporting accuracy by strata, the stratified design

254 allows specifying a sample size for each stratum to ensure that a precise estimate is obtained for

255 each stratum. Land change often occupies a small proportion of the landscape, so a change

256 stratum can be identified and the sample size allocated to this stratum can be large enough to

257 produce a small standard error for the change user's accuracy estimate.

258 The practical reality is that limited resources will likely be available for the reference sample

259 and this constraint will strongly impact sample allocation decisions because different allocations

260 favour different estimation objectives. For example, allocating equal sample sizes to all strata

261 favours estimation of user's accuracy over estimation of overall and producer's accuracies

262 (Stehman, 2012). Conversely, the standard errors for estimating producer's and overall

263 accuracies are typically smaller for proportional allocation (i.e., the sample size allocated to each

264 stratum is proportional to the area of the stratum) relative to equal allocation. As a compromise

265 between favouring user's versus producer's and overall accuracies, the allocation recommended

266 is to shift the allocation slightly away from proportional allocation by increasing the sample size

267 in the rarer classes, but the sample size for the rare classes should not be increased to the point

268 where the final allocation is equal allocation (see Section 5 for an example). The sample size

269 allocation decision can be informed by calculating the anticipated standard errors (see Sections

2704.3 and 4.4) for different sample sizes and different allocations. An ineffective allocation of

271 sample size to strata will not result in biased estimators of accuracy or area, but it may result in

272 larger standard errors (see Section 5 for an example). 
When stratified sampling is applied to a single date land-cover map, it is usually feasible to

274 define a stratum for each land-cover class (Wulder et al., 2007). Identifying an effective

275 stratification for change can be more challenging. A common approach is to use a map of change

276 to identify the strata, and such strata are effective for estimating user's accuracy of change

277 precisely. However, the number of different types of change may be so large that defining every

278 change type as a stratum is not advisable. For example, in a post-classification comparison of

279 two land-cover maps, that each include with a map legend that includes-8 land-cover classes,

280 there are 56 possible types of change in the final change map. If each stratum must receive a

281 relatively large sample to achieve a precise user's accuracy estimate, the overall sample size may

282 be unaffordable.

283 The trade-offs between precision of user's accuracy, producer's accuracy, and area estimates

284 from different sample size allocations become exacerbated as the number of strata increases.

285 Some types of change may be very unlikely to occur and consequently could be eliminated as

286 strata. To further reduce the number of strata, strata could be defined on the basis of generalized

287 change categories (Wickham et al., 2013). For example, a stratum could be change from any

288 class to urban (i.e., urban gain), and another stratum could be change to any class from forest

289 (i.e., forest loss). These generalized or aggregated change strata are obviously less focused on all

290 possible individual change types. For example, the forest loss stratum could include forest to

291 developed, forest to water, or forest to cropland. These generalized change strata would allow for

292 specifying the sample size allocated to different general change types, but within one of the

293 generalized strata, the sample size allocated to the individual change types would be proportional

294 to the area of that change type. For example, if the most common type of forest loss is to

295 cropland and the least common change is forest loss to water, many more of the sample units 
within the forest loss stratum will be forest-to-cropland-conversion. Strahler et al. (2006, Fig.

297 5.2, p. 32) provides additional examples of aggregated change classes that could be used as 298 strata.

299 The desire to limit the number of strata motivates discussion of subpopulation estimation as it 300 relates to sampling design. A subpopulation is any subset of the ROI, for example a particular 301 type of change or a particular subregion. Subpopulations can be defined as strata, but it is not 302 necessary for a subpopulation to be defined as a stratum to produce an estimate for that 303 subpopulation. For example, when aggregating multiple types of change into a generalized 304 change stratum, it would still be possible to estimate accuracy of each of the subpopulations 305 representing the individual types of change making up the aggregated change stratum.

306 However,But if these subpopulations are not defined as strata, the sample size representing the 307 subpopulation may not be large enough to obtain a precise estimate. Resources available for 308 accuracy assessment may require limiting the number of strata used in the design, so prioritizing 309 subpopulations may be necessary to establish which subpopulations are defined as strata.

310 It is sometimes the case that several maps will be assessed based on a common accuracy

311 assessment sample. This forces a decision on whether the strata should be based on a single map

312 (and if so, which map) or if the strata should be defined by a combination of the multiple maps.

313 Once strata are defined and the sample is selected using these strata, the strata become a fixed

314 feature of the design because the analysis is dependent on the estimation weight associated with

315 each sample unit and this weight is determined by the sampling design. Fortunately, whatever the

316 decision is to define strata when multiple maps are to be assessed, the sample reference data are

317 still valid to assess any of the maps, even if the strata are defined on the basis of a single map.

318 The principles of estimation outlined in the Analysis Section (Section 4) must be adhered to, and 
319 this simply requires using the estimation weights for the sample units determined by the original

320 stratified selection protocol. The impact of the choice of strata will be reflected in the standard

321 errors of the estimates. Olofsson et al. (2012) and Stehman et al. (2012) discuss sampling design

322 issues associated with constructing a reference validation database that would allow assessment

323 of multiple maps.

324 To summarize the recommendations related to the important question of whether to 325 incorporate stratification in the sampling design, stratifying by mapped change and by

326 subregions is justified to achieve the objective of precise class-specific accuracy and to report

327 accuracy by subregion. If the overall sample size is not adequate to support both class-specific

328 and subregion accuracy estimates, the subregional stratification may be omitted and accuracy by

329 subregion relegated to the status of subpopulation estimation. The recommended allocation of

330 sample size to the strata defined by the map classes is to increase the sample size for the rarer

331 classes making the sample size per stratum more equitable than what would result from

332 proportional allocation, but not pushing to the point of equal allocation. The rationale for this

333 recommendation is that user's accuracy is often a priority objective and we can control the

334 precision of the user's accuracy estimates by the choice of sample allocation. However, the

335 trade-off is that a design allocation chosen solely for the objective of user's accuracy precision

336 (i.e., equal allocation) may be detrimental to precision of estimates of overall accuracy,

337 producer's accuracy, and area, so a compromise allocation is in order. Lastly, defining

338 aggregations of change types as strata may be necessary if the number of strata needs to be

339 limited, and accuracy and area estimates for the individual change types would be obtained as

340 subpopulation estimates. 


\subsubsection{Cluster Sampling}

342 A cluster is a sampling unit that consists of one or more of the basic assessment units specified

343 by the response design. For example, a cluster could be a 3 x 3 block of 9 pixels or a $1 \mathrm{~km} \mathrm{x} 1$

$344 \mathrm{~km}$ cluster containing 1001 ha assessment units. In cluster sampling, a sample of clusters is

345 selected and the spatial units within each cluster are therefore selected as a group rather than

346 selected as individual entities. Each of the spatial units within a cluster is still interpreted as a

347 separate unit even though it is selected into the sample as part of a cluster. For example, a $3 \times 3$

348 pixel cluster would require obtaining the reference classification for individual pixels within the

349 cluster.

350 The primary motivation for cluster sampling is to reduce the cost of data collection. For 351 example, if field visits are required to obtain the reference classification, transit time and costs

352 may be reduced if the sample units are grouped spatially into clusters. Zimmerman et al. (2013)

353 used cluster sampling to reduce the number of raster images (i.e., clusters) required because the

354 primary cost of the sampling protocol was associated with processing the very high resolution

355 images used for reference data. As another example, Stehman and Selkowitz (2010) used a 27

$356 \mathrm{~km} \times 27 \mathrm{~km}$ cluster sampling unit to constrain sample locations to a single day of flight time per

357 cluster when the reference data were collected by aircraft. Cluster sampling may also be

358 motivated by the objectives of an accuracy assessment. For example, a cluster sampling unit

359 becomes necessary to assess accuracy at multiple spatial supports (e.g., single pixel, 1 ha unit, 360 and $1 \mathrm{~km}^{2}$ unit).

361 The cost savings gained by cluster sampling should be substantial before choosing this 362 design because the correlation among units within a cluster (i.e., intracluster correlation) often 363 reduces precision relative to a simple random sample of equal size. Focusing on the specific 
364 example of estimating land-cover area in Europe, Gallego (2012) showed that a $10 \mathrm{~km}$ x $10 \mathrm{~km}$

365 sampling unit produced equivalent information to that of a simple random sample of only 25

366 points or fewer. The low yield of information per cluster diminishes the cost advantage of

367 cluster sampling if the intracluster correlation is high. Another potential disadvantage of cluster

368 sampling is that it complicates stratification when the strata are the map classes and the

369 assessment unit is a pixel. In the simplest setting, each cluster would be assigned to a stratum,

370 but rules have to be established for assigning a cluster to a stratum when the cluster includes area

371 of several different classes. Cluster sampling can be combined with stratification of pixels by the

372 map class of each pixel in a two-stage stratified cluster sampling approach (Stehman et al., 2003,

373 2008), but such designs require more complex analysis and implementation protocols than what

374 are required of a stratified design without clusters. Because of the added complexity $\theta$ fluster

375 sampling introduces for sampling design (e.g., accommodating stratification within a cluster

376 sampling design) and estimation (e.g., estimating standard errors), we recommend this design

377 only in cases for which the objectives require a cluster sampling unit or in which the cost savings

378 or practical advantages of cluster sampling are substantial.

\section{2.1.3. Systematic vs. Random Selection}

380 The two most common selection protocols implemented in accuracy assessment are simple

381 random and systematic sampling (we define "systematic" as selecting a starting point at random

382 with equal probability and then sampling with a fixed distance between sample locations). Both

383 protocols can be implemented to select units from within strata or to select clusters, and both can

384 be applied to a ROI that is not partitioned into strata or clusters. Unbiased estimators of the

385 various accuracy parameters are available from either systematic or simple random selection, so

386 the bias criterion is not a basis for choosing between these options. Instead, the choice of simple 
random versus systematic depends on how each selection protocol satisfies the priority desirable

388 design criteria (Stehman, 2009). For example, systematic sampling is often simpler to implement

389 when the response design is based on field visits, but the greater convenience of systematic

390 versus simple random is diminished when working with imagery or aerial photographs as a

391 source of the reference data. Typically ${ }_{2}$ systematic selection will yield more precise estimates

392 than simple random selection, but systematic sampling requires use of a variance approximation

393 so if unbiased variance estimation is a priority criterion, simple random is preferred. Simple

394 random selection also is advantageous if it is likely that the sample size will need to be modified

395 during the course of the accuracy assessment (Stehman et al., 2012). A scenario in which

396 systematic selection opportunistically arises is when accuracy assessment reference data can be

397 simultaneously obtained in conjunction with another field sampling activity. For example, many

398 national forest inventories employ a systematic sample of field plots (Tomppo et al., 2010) and

399 these field plot data may be an inexpensive, high quality source of reference data. In general, the

400 simple random selection protocol will better satisfy the desirable design criteria and is the

401 recommended option. However, systematic selection is also nearly always acceptable.

\section{2.2. A Recommended Good Practice Sampling Design}

403 Stratified random sampling is a practical design that satisfies the basic accuracy assessment 404 objectives and most of the desirable design criteria. Stratified random sampling affords the 405 option to increase the sample size in classes that occupy a small proportion of area to reduce the 406 standard errors of the class-specific accuracy estimates for these rare classes. Thus this design 407 addresses the key objective of estimating class-specific accuracy. In regard to the desirable 408 design criteria, stratified random sampling is a probability sampling design and it is one of the 409 easier designs to implement. Stratified sampling is commonly used in accuracy assessment so it 
410 has an advantage of being familiar to the remote sensing community (cf. Mayaux et al., 2006;

411 Cakir et al., 2006; Huang et al., 2010; Olofsson et al., 2011). Increasing or decreasing the sample

412 size after the data collection has begun is readily accommodated by stratified random sampling,

413 and unbiased variance estimators are available thus avoiding the need to use variance

414 approximations. An assumption implicit in this recommendation is that change between two

415 dates is of interest. Little work has been done to investigateing the effective use of strata for

416 multiple change periods. Stratifying by a change map also assumes that it is possible to obtain

417 the reference data for the initial date of the change period given that the change map will not be

418 available until the end date of the change period. If this is not possible, stratification is still an

419 option but the strata would need to be constructed on the basis of predicted change.In the case of

420 stratification based on a change map, it is assumed that reference data for the sampled locations

421 exists for the initial date of the change period (e.g., archived imagery or aerial photography is

422 available). If the reference data must be obtained in real time (e.g., via ground visit), it would not

423 be possible to stratify by a change map that does not yet exist at the initial date. An alternative

424 would be to stratify by anticipated change or predicted change, with the effectiveness of such

425 strata dependent on how well the predicted change matched with the ensuing reality of change.

\section{3. Response Design}

427 For the accuracy assessment objective, the response design encompasses all steps of the protocol

428 that lead to a decision regarding agreement of the reference and map classifications. For area

429 estimation, the response design provides the best available classification of change for each

430 spatial unit sampled. The Ffour major features of the response design are the spatial unit, the

431 source or sources of information used to determine the reference classification, the labelling 
432 protocol for the reference classification, and a definition of agreement. Each of these major

433 features is discussed in the following subsections.

\section{3.1. Spatial Assessment Unit}

435 The spatial unit that serves as the basis for the location-specific comparison of the reference

436 classification and map classification can be a pixel, polygon (or segment), or block (Stehman and

437 Wickham, 2011). The ROI is partitioned based on the chosen spatial unit (i.e., the region is

438 completely tiled by these non-overlapping spatial units). Commonly, the pixel is selected as the

439 spatial unit. The pixel is an arbitrary unit defined mainly by the properties of the sensing system

440 used to acquire the remotely sensed data or a function of the grid used to sub-divide space in a

441 raster based data set. A polygon is defined as a unit of area, perhaps irregular in shape,

442 representing a meaningful feature of land cover. For example, a polygon may be delineated from

443 a map such that the area within the polygon has the same map classification (e.g., the entire

444 polygon is stable forest or the entire polygon represents an area of change from forest to urban).

445 Polygons defined on the basis of a map will be called "map polygons." Alternatively, a polygon

446 could be delineated on the basis of the reference classification as an area within which the

447 reference class is the same. A polygon delineated on the basis of the reference classification will

448 be called a "reference polygon". A "block" spatial assessment unit is defined as a rectangular

449 array of pixels (e.g., a $3 \times 3$ block of pixels). Irrespective of the spatial unit selected, it is

450 important to note that some spatial units may be impure, that isi.e., they represent an area of

451 more than one class. Mixed pixels are, for example common, especially in coarse spatial

452 resolution data. Similarly, it is, for example, possible that a map polygon is not internally

453 homogeneous in terms of the reference classification, and a reference polygon may not be

454 internally homogeneous in terms of the map classification. A polygon defined by a segmentation 
455 algorithm would not necessarily be homogeneous in terms of either the map or the reference 456 classifications.

457 Pixels, polygons, or blocks can be used as the spatial unit in accuracy assessment.

458 Regardless of the unit chosen, a critical feature of the response design protocol is that the

459 spatially explicit character of the accuracy assessment must be retained. Practitioners should aim

460 to have reference data with an equal or finer level of detail than the data used to create the map,

461 but we make no recommendation is made regarding the choice of spatial assessment unit.

462 However, once the spatial assessment unit has been chosen, there will be good practice

463 recommendations associated with that specific unit and the choice of spatial unit also has

464 implications on the sampling design (Stehman and Wickham, 2011) and analysis. Estimates of

465 accuracy and area derived from the same map but through the use of different spatial units may 466 be unequal.

\section{3.2. Sources of Reference Data}

468 The reference classification can be determined from a variety of sources ranging from actual

469 ground visits to the sample locations or the use of aerial photography or satellite imagery. There

470 are two ways to $\mathrm{Fe}$ ensure that the reference classification is of higher quality than the map

471 classification: either the reference source has to be of higher quality than what was used to

472 create the map classification, and 2) 1 r if using the same source material for both the map and

473 reference classifications, the process to create the reference classification has to be more accurate

474 than the process used to create the classification being evaluated. (e.g.For example, if Landsat

475 imagery is used to create the map and Landsat is the only available imagery for the accuracy

476 assessment, then the process for obtaining the reference classification has to be more accurate

477 than the process for obtaining the map classification-. FurtherAdditionally, other spatial data may 
478 be used to improve the quality of the reference classification, such as forest inventory data or

479 some form of vector data (e.g., roads, pipelines, or crop records). In this subsection, different

480 potential sources of reference data for assessing accuracy of change are identified and strengths

481 and weaknesses of these sources are described.

482 Possible reference data sources include field plots, aerial photography, forest inventory data,

483 airborne video, lidar, and satellite imagery (Table 1). Additional sources of freely accessible

484 reference data may also be opportunistically available from data mining and crowdsourcing

485 (Iwao et al., 2006; Foody and Boyd, 2013). and silvicultural records (Hyyppä et al., 2000;

486 Wulder et al., 2006a).

$<<$ TABLE 1 HERE $>>$

490 Practical considerations regarding costs often influence the selection of reference data, or the use 491 of existing data. While existing or lower cost data may be desirable from a purchase perspective, 492 the use of disparate data sources will result in additional effort by project analysts to deal with 493 exceptions and inconsistencies. A key to using disparate data sources is to have the reference 494 data that are actually used in the accuracy assessment be, as much as possible, invariant to 495 source. For example, the creation of attributed change polygons makes the polygon the common 496 denominator, rather than the source data. Creating polygonal change units in a portable format 497 and populating a minimum set of fields to support a consistent labelling protocol is desirable. 498 The information to be recorded for each change unit is itemized in Table 2. 
502 Ideally a data source is available for the entire with uniform likelihood over the-ROI,

503 representing the change types and dates of interest, at a low cost. The realities versus the ideal

504 result in a series of considerations are detailed in Table 3. For instance, if the ROI is small, the

505 costs may be less of an issue and access may not be relevant. For large area projects over poorly

506 monitored areas, existing data sources are not often available so data purchase and interpretation

507 costs become the dominant criteria. The ease of interpretation and consistency of source

508 reference data permits economies in the project flow for the analysts and also promotes

509 automation of repeated activities. Further, the development of a well documented and consistent

510 change validation data set will have utility for multiple projects and purposes.

\section{$<<$ TABLE 3 HERE $>>$}

514 Both high- and very high spatial resolution satellite data are viable candidates for reference data.

515 Imagery is typically considered as very high spatial resolution (VHSR) with a spatial resolution

516 of when pixels are sided $<1 \mathrm{~m}$ and high spatial resolution (HSR) with a spatial resolution of $<10$

$517 \mathrm{~m}$. Both data sources provide information that is finer than the data used in most large area

518 monitoring projects, which would typically have imagery with a spatial resolution of greater

519 than $10 \mathrm{~m}$. At the fine spatial resolution of satellite-borne VHSR imagery, panchromatic is often

520 the only spectral information collected. The typical 400 to $900 \mathrm{~nm}$ panchromatic data with small

521 pixels ( $0.50 \mathrm{~m}$ in the case of WorldView-1) closely resemble large scale aerial photography and

522 can be interpreted using established aerial photograph interpretation techniques (Wulder et al.,

523 2008a) or subject to digital analyses (cf. Falkowski et al., 2009). Both the SPOT Image ${ }^{\circledR}$ and 
524 DigitalGlobe ${ }^{\circledR}$ archives can be accessed through Google Earth ${ }^{\mathrm{TM}}$, with the image extents by year 525 portrayed. The presence of freely accessible high spatial resolution imagery online, freely 526 accessible, through Google Earth ${ }^{\mathrm{TM}}$ also presents low cost interpretation options. Limitations of 527 this approach include a lack of data prior to the initiation of the high spatial resolution satellite 528 commercial era (circa 2000), spatial distribution of available imagery, and the actual temporal 529 revisit of the images available. The reported temporal revisit can be on the order of days based 530 upon an ability to point the sensor head. For instance, IKONOS has off-nadir revisit of 3 to 5

531 days, with 144 days required for nadir revisit (Wulder et al. 2008b). The implication is that when

532 the sun-surface-sensor viewing geometry changes the structure captured changes, such that trees

533 evident on one image may be occluded in another. For a given on-line accessible source of

534 satellite imagery, it should not be expected that historical, archival, global coverage from launch

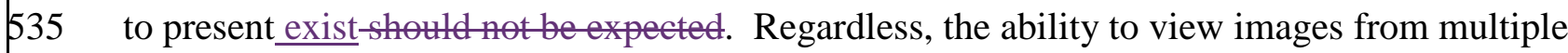
536 years can help determine that date when a change (e.g., a disturbance) occurred. The additional 537 context provided around particular change events aids with interpretation of change type (e.g., 538 determination of harvesting versus forest removal in support of agricultural expansion).

539 Development and sharing of a change data base, once interpreted and attributed following 540 defined procedures, leveraging Google Earth ${ }^{\mathrm{TM}}$ is a consideration for global or large area 541 aecuracy assessment aetivities.

542 There are few, if any, reference data sources that are available with a uniform likelihood 543 globally. There are some archival datasets with wide global coverage (e.g., Kompsat); although, 544 the utility of these data sets may be limited. The utility of any given datareference data source 545 when used to capture and relate change is the date or represented by vintage of the data. While 546 less of an issue with satellite data, air photos and maps may not be of a known vintage. 
547 Acquisition dates of historic photos are often lost, plus maps are often representative of a period,

548 not a singular date. Knowing the conditions that previously existed may not be helpful if the date 549 of change occurrence is not known.

550 Over some regions, land use change and silvicultural records may also be available to inform 551 on the land_-cover change. Note that forest harvesting is a land--cover change relating a 552 successional stage, rather than a land use change (which implies a permanent change in how a 553 particular parcel of land is used - e.g., forestry to agriculture). The-This distinction is important 554 for both monitoring and reporting purposes as the permanent removal of forests has differing 555 carbon consequences than a forest harvesting (Kurz, 2010).

556 While the good practice guidelines advocate for use of reference data of finer spatial

557 resolution than the map product, this is especially so for single date interpretations of the 558 reference data. Following the opening of the Landsat archive by the USGS (Woodcock et al., 559 2008), time series of imagery ereates-created new opportunities for using imagery of the same 560 spatial resolution (e.g., Landsat) when archival data are available. Simple visual approaches may

561 be applied, such as in Figure 1, where a change event (fire) that is evident in 2010 can be timed 562 quite precisely by the evidence captured (smoke plume) showing when the fire is-occurreding.

563 This type of change dating is rather opportunistic and not to be commonly expected.

<<FIGURE 1 HERE $>$

567 Figure 1. Landsat data can be used for the visual dating of change, with the fire event in progress

568 in Inset A, August 3, 2010, with the burned forest outcome evident in Inset B, September 20, 569 2010, Yukon, Canada (Landsat Path 55, Row 18). 
571 A more reliable means for determining the timing of change events can be from developing 572 and interrogating time series of images (Kennedy et al., 2010). To ensure the quality of time 573 series transitions developed, Cohen et al. (2010) created a logic and tool for determining the 574 timing and nature of changes captured (TimeSync, http://timesync.forestry.oregonstate.edu/).

575 Based upon the image collection and archiving protocols present through the history of Landsat, 576 the spatial and temporal coverage of imagery is not uniform. The temporal precision possible for 577 dating changes based upon time series analysis is likely weaker for locations that already have a 578 paucity of data. This situation is due to the historic practices followed at given Landsat receiving 579 stations through to the commercial era (during the 1980s) when fewer images were collected and 580 archived (Wulder et al., 2012). It should not be assumed that the temporal density possible for 581 the conterminous United States is possible for all other regions (Schroeder et al., 2011).

582 Another critical aspect of the response design is that the change period represented by the 583 reference classification must be synchronous with the change period of the classification.

584 Consider a map representing change between 2000 and 2010. To capture near anniversary dates 585 (within year) and athe northern hemisphere peak photosynthetic period, the imagery used for this 586 hypothetical project was collected July 15, 2000, and 10 years later, July 15 2010. The reference 587 data should be collected in 2010, but ideally not after July 15 (assuming similar satellite overpass 588 times) to avoid confusion. Data collected after July 15, 2010 will have to be vetted to ensure the 589 change present in the reference data did not occur after the product date of the change map. 590 Imagery from the same year is desired but may not always be possible. As such, it is required 591 that the change reference data includes-approximates the date the change occurred as precisely as 592 pessibleavailable. Multiple images help refine the timing of the change event. Mismatched 
593 change periods between the map and reference classifications would be a major source of

594 reference data error.

\section{3.3. Reference Labelling Protocol}

596 The labelling protocol refers to the steps in the response design that take the information

597 provided by the reference data and convert that information to the label or labels constituting the

598 reference classification. Labelling is far from trivial with numerous definitions for land--cover

599 classes in use (cf. Comber et al., 2008 ) although recent developments such as the FAO's Land

600 Cover Classification system (LCCS) may act to enhance interoperability (Ahlqvist, 2008). The

601 labelling protocol should also include specification of a minimum mapping unit (MMU) for the

602 reference classification. The MMU can have important implications for accuracy assessment and

603 area estimation. For example, increasing the size of the MMU will lead to a reduction in the

604 representation of classes that occupy small, often fragmented, patches (Saura, 2002). Changing

605 the MMU can also impact en-accuracy estimates, although the effect is most apparent when a

606 large change is made (Knight and Lunetta, 2003). Clearly, s్mall patches present a challenge to

607 mapping (cf. He et al., 2011) and the accuracy of their mapping will degrade as the MMU is

608 increased. However, but it is possible that overall map accuracy may increase with a larger

609 MMU, making it is important to ensure that attention is focused on an appropriate measure of

610 accuracy for the application in-hand. The precise effects of the MMU will vary as a function of

611 the land--cover mosaic under study and the imagery used. The MMU specified for the response

612 design does not necessarily have to match the MMU specified for the map. In fact, if the

613 reference classification is intended to apply to a variety of maps, it would be likely that the

614 MMU of the reference classification does not match the map classification for all maps that

615 might be assessed. Often the reference imagery or information will permit distinguishing smaller 
616 patches or features than can be distinguished from the map so a smaller MMU will be possible

617 for the reference classification.

618 The easiest case for the labelling protocol occurs when the assessment unit is homogeneous

619 and a single reference class label can be assigned (the reference class could be a type of change).

620 But $\oplus$ Often, however, the situation will be more complex making class labelling less certain. For

621 example, the assessment unit may contain a mixture of classes, and even if the unit is

622 homogeneous, it may be difficult to assign a single label (e.g., change type) because the unit is

623 not unambiguously one of the classes in the legend but instead falls between two of the discrete

624 class options in the legend (i.e., land_-cover classes are a continuum represented on a discrete

625 scale). A variety of options exist for labelling a unit when a single reference label does not

626 adequately represent the uncertainty of a unit. One or more alternate reference class labels can be

627 assigned to account for ambiguity in the reference classification. Another option when defining

628 agreement is to construct a weighted agreement based on how closely the different classes are

629 related. For example, in the GlobCover assessment, a "matrix" of class relationships was

630 established (Mayaux et al., 2006, GLC2000). A fuzzy reference labelling protocol may also be

631 employed, for examplesuch as the linguistic scale devised by Gopal and Woodcock (1994) or a

632 fuzzy membership vector in which the reference label for a unit specifies a membership value for

633 each class (Foody, 1996; Binaghi et al., 1999). Another option for mixed units is to specify the

634 proportion of area of each class present in the unit (Foody et al., 1992; Lewis and Brown, 2001).

635 A different characterization of uncertainty in the reference classification is obtained by assigning

636 a confidence rating that represents the interpreter's perception of uncertainty in the reference

637 classification for that unit. For example, low, moderate and high confidence ratings would

638 indicate increasing confidence on the part of the interpreter that the reference classification is 
correct. Typically this information can then be used in the analysis to subset results by

640 confidence rating (Powell et al., 2004; Wickham et al., 2001, Table 4).

641 The response design should include protocols to enhance consistency of the reference class

642 labelling. For example, interpretation keys should be created if visual assessment is used to

643 obtain the reference classification (Kelly et al., 1999) and specific instructions to translate

644 quantitative field data into reference labels should be provided and documented. If multiple

645 interpreters are used, training interpreters to ensure consistency is critical. Interpreters should be

646 in communication throughout the process to discuss and review difficult cases and to agree upon

647 a common approach to labelling such cases. Difficult cases should be noted for future reference

648 and consensus development (e.g., the imagery is retained and accessible, and the decision

649 process leading to the reference label of the case is documented). Rather than solely visual

650 approaches, entire high spatial resolution images can be classified, with the underlying imagery

651 also maintained and accessible as support information to the accuracy assessment (that is, to

652 gain/ensure confidence in the categories selected for a given location).

\section{3.4. Defining Agreement}

654 Once the map and reference classifications have been obtained for a given spatial unit, rules for 655 defining agreement must be specified before proceeding to the analyses that quantify accuracy.

656 In the simplest case, a single class label is present for the map and a single label is provided by

657 the reference classification. If these labels agree, the map class is correct for that unit, $;$ and if the 658 labels disagree, the type of misclassification is readily identified. Defining agreement becomes

659 more complex if the assessment unit is not homogeneous or if more than a single one class label 660 is assigned by the map or reference classification. For example, if the reference classification

661 provides a primary and secondary reference label, agreement can be defined as a match between 
662 the map label and either the primary or secondary reference label. If the reference classification

663 consists of a vector of proportions of area of the classes present in the assessment unit (e.g., the

664 area proportions of the classes are $0.2,0.5$, and 0.3 ), agreement can be defined as the proportion

665 of area for which the map and reference labels are the same. The critical feature of the protocol

666 for defining agreement is that it allows construction of an error matrix in which the elements of

667 the matrix represent proportion of area of agreement and disagreement between the map and

668 reference classifications. These proportions (in terms of area) achieve the necessary spatially

669 explicit assessment of map accuracy and the requirements for area estimation.

\section{3.5. Reference Classification Uncertainty: Geolocation and Interpreter Variability}

671 In an ideal case, the reference classification is based on a reference data set of such quality that

672 the sample labels represent the ground truth (i.e. a "gold standard" reference data set). However,

673 the reference classification is subject to uncertainty, and an assessment of this uncertainty should

674 be conducted. Small errors in the reference data set can lead to large biases of the estimators of

675 both classification accuracy and class area (Foody, 2010; 2013). Two potential sources of

676 uncertainty in the reference classification are the uncertainty associated with spatial co-

677 registration of the map and reference location (Pontius, 2000) and uncertainty associated with the

678 interpretation of the reference data (Pontius and Lippitt, 2006).

679 Geolocation error is defined as a mismatch between the location of the spatial assessment

680 unit identified from the map and the location identified from the reference data. The response

681 design should be constructed to minimize geolocation error. For instance, it is common for plots

682 to have a GPS position. The quality of the GPS position can be related by to the type of

683 instrument used, which can provide an indication of spatial precision. The length of time,

684 number of position measures to resolve the location, and the number of satellites are also aspects 
685 that can be recorded. The magnitude of geolocation error should be characterized by

686 documenting the spatial location quality of the map and reference data sources (e.g., GPS units,

687 aerial photography, or satellite imagery). If airborne imagery is to be used, aircraft positioning

688 and pointing information should be collected. The GPS location of the aircraft does not

689 necessarily indicate the position of the point on the ground that is captured in photographic or

690 video data. A slight roll of the aircraft can create a mismatch between the recorded and actual

691 positions. Error in the classification may be incorrectly indicated due to these spatial

692 mismatches, especially for smaller change events or rare classes.

693 Interpreter uncertainty can be separated into two parts: 1) interpreter bias is defined as an

694 error in the assignment of the reference class to the spatial unit; 2) interpreter variability is a

695 difference between the reference class assigned to the same spatial unit by different interpreters

696 (i.e., interpreter variability is the complement of among interpreter agreement). Although iddeally

697 an assessment of both interpreter bias and interpreter variability would be conducted;-;in

698 practice, assessing only interpreter variability may be feasible. The difficulty hindering

699 assessment of interpreter bias is whether a "gold standard" of truth exists against which the

700 interpreted reference classification can be compared. For example, on-the-ground reference data

701 may serve to establish the gold standard of truth for land cover at a single date, but a gold

702 standard for change based on field visits would be much more difficult and costly to establish.

703 Comparison of interpreters to an "expert" interpreter is a practical but less satisfying option for

704 quantifying interpreter bias and the success of this approach depends on how closely the expert

705 classification mimics the gold standard. A distinction between the accuracy assessment of land

706 cover and change does exist, whereby the continuous nature of land cover benefits more from

707 field visits. Depending on the change categories of interest, field visits may not be as 
708 informative. For example, slower continuous changes may benefit from field visits, but rapid

709 stand replacing disturbances may not. The date of change, if not captured in silvicultural records

710 or fire maps, may actually be better captured from imagery of known vintage than through field

711 visits (Cohen et al., 2010).

712 If multiple interpreters or interpreter teams are providing the reference classification,

713 interpreter variability can be assessed by having interpreters classify a common sample of

714 locations. Ideally, the sample would include locations covering a variety of classes to allow

715 evaluating how interpreter variability differs by class (e.g., do interpreters consistently agree for

716 some classes, but not others). The quality of the interpreters in terms of the accuracy of their

717 tabelling may also be assessed directly from the data generated (Foody et al., 2013). If this

718 evaluation sample is selected using a probability sampling design (see Section 2), estimates of

719 interpreter variability will have a strong inferential basis and results from the sample can be

720 rigorously inferred to the population of all interpretations. If multiple interpreters operating

721 independently are employed to determine the reference classification for each sample location, a

722 number of considerations may affect the decision of how many interpreters are used. Wulder et

723 al. (2007) who used seven interpreters in a land cover labelling protocol, detail the issues that

724 arise when using multiple interpreters, noting common disagreement between interpreters,

725 especially for more refined and rare classes. Ensuring that consensus is reached, rather than an

726 aggregation of independent interpretations, is also possible. Also using airborne video data,

727 Powell et al. (2004) required five interpreters to agree upon a specific class, with the outeome

728 then treated as a "gold standard". While some disagreement could be linked to difficulty in

729 identifying the vegetation in the video, other sources of disagreement included data entry error

730 and misreading of sample labels. These are sources of error that can be mitigated by using 
731 intelligent data management and entry tools. Wulder et al. (2007), recommend the use of an
732 independent evaluation protocol, followed by cross-calibration, and the revisit of problematic
733 ef multiple interpreters.
$735 \quad$ A number of issues arise when using multiple interpreters to obtain the reference
$736 \quad$ classification (Wulder et al. 2007). Disagreements among interpreters evaluating the same
$738 \quad$ sampling unit are likely. These disagreements may be resolved by a consensus agreement on the
$739 \quad$ specific class, with the outcome then treated as a "gold standard". Constant communication
$740 \quad$ among the multiple interpreters to discuss and document difficult cases is important to foster
$742 \quad$ enhanced consistency and accuracy of the reference labeling process (Wickham et al. 2013).
$743 \quad$ The response design protocols described in this section have has-focused on land--cover

743 changes that can be characterised by a complete change in class type: conversions of cover. In

744 some studies attention is focused on more subtle changes or modifications of land cover, as

745 changes in land cover can be considered as processes (Gomez et al., 2011) with depletions-gains

746 and accruals-losses in vegetation captured and possible to assign a label (Kennedy et al., 2010).

747 Cohen et al. (2010) show how investigation of time series of satellite imagery supported by

748 period photography can illuminate en-subtle changes in forest conditions (-such as decline due to

749 insects or water stress and conversely recovery of forests following disturbance- - The impertance

750 of the ability to capture and label subtle changes is dependent upon the goals of the change

751 elassification. The interest in quantifying emissions of $\mathrm{CO}_{z}$ to the atmosphere, a full accounting

752 of subtle changes is increasingly desired, with capture of degradation (FAO, 2011) while

753 difficult of interest for averting and related documentation of deforestation (UN REDD, 2008). 
754 The response design protocols presented also do not address the situation in which the map

755 provides information as a continuous variable. Although many of the basic concepts underlying

756 the good practice recommendations would apply to a continuous variable, the details of

757 methodology of the accuracy assessment methodology (cf. Riemann et al., 2010) and area

758 estimation would likely be considerably different from the methods presented herein.

\section{4. Analysis}

760 The analysis protocol specifies the measures to be used to express accuracy and class area as

761 well as the procedures to estimate the selected measures from the sample data-aequired. In the

762 context of studies of land change, there are two key objectives of the analysis: 1) accuracythe

763 assessment $\theta$ the accuracy of the change classification, and 2) estimation the provision of

764 information on the-area of change. The confusion or error matrix (hereafter noted as the error

765 matrix) plays a central role in meeting both the accuracy assessment and area estimation

766 objectives (Foody, 2013; Stehman, 2013).

\section{$767 \quad$ 4.1 The Error Matrix}

768 The error matrix is a simple cross-tabulation of the class labels allocated by the classification of

769 the remotely sensed data against the reference data for the sample sites. The error matrix

770 organizes the acquired sample data in a way that summarizes key results and aids the

771 quantification of accuracy and area. The main diagonal of the error matrix highlights correct

772 classifications while the off-diagonal elements show omission and commission errors. The cell

773 entries and marginal values of the error matrix are fundamental to both accuracy assessment and

774 area estimation. Table 4 illustrates a four-class example error matrix of the type often used in

775 studies of land change. 
779 The rows of the error matrix represent the labels shown in a map derived from the classification

780 of the remote sensing data and the columns represent the labels depicted in the reference data.

781 This layout is not a universal requirement and some may wish to reverse the contents of the rows

782 and columns. In the matrix, $p_{i j}$ represents the proportion of area for the population that has map

783 class $i$ and reference class $j$, where "population" is defined as the full region of interest, and $p_{i j}$ is

784 therefore the value that would result if a census of the population were obtained (i.e., complete

785 coverage reference classification).

786 Accuracy parameters derived from a population error matrix of $q$ classes include overall

787 accuracy

788

$789 \quad O=\sum_{j=1}^{q} p_{j j}$

791 user's accuracy of class $i$ (the proportion of the area mapped as class $i$ that has reference class $i$ )

792

$793 U_{i}=p_{i i} / p_{i}$

794

795 or its complementary measure, commission error of class $i, 1-p_{i i} / p_{i}$, and producer's accuracy

796 of class $j$ (the proportion of the area of reference class $j$ that is mapped as class $j$ ),

797

$798 \quad P_{j}=p_{j j} / p_{\cdot j}$ 
800 or its complementary measure, omission error of class $j, 1-p_{j j} / p_{\cdot j}$. A variety of other measures

801 of accuracy has been used in remote sensing (Liu et al., 2007). A commonly used measure is the

802 kappa coefficient of agreement (Congalton and Green, 2009). The problems associated with

803 kappa include but are not limited to: 1) the correction for hypothetical chance agreement

804 produces a measure that is not descriptive of the accuracy a user of the map would encounter

805 (kappa would underestimate the probability that a random selected pixel is correctly classified);

806 2) the correction for chance agreement used in the common formulation of kappa is based on an

807 assumption of random chance that is not reasonable because it uses the map marginal proportions

808 of area in the definition of chance agreement and these proportions are clearly not simply

809 random; and 3) kappa is highly correlated with overall accuracy so reporting kappa is redundant

810 with overall accuracy."However, kappa has numerous problems not least an incorrect and

811 unnecessary “correction” for chance agreement (Foody, 1992; Stehman, 1997; Liu et al., 2007;

812 Pontius and Millones, 2011). Consistent with the recommendation in Strahler et al. (2006), the

813 use of kappa is strongly discouraged as, despite its widespread use, it actually does not serve a

814 useful role in accuracy assessment or area estimation.

\section{$815 \quad$ 4.2 General Principles of Estimation for Good Practice}

816 The eore nature of the analysis protocol is designed to achieve the objectives of estimating

817 produce estimates of accuracy and area from the sample data. Analysis thus requires statistical

818 inference as the underlying scientific support for generalizing from the sample data to the

819 population parameters and for quantifying uncertainty of the sample-based estimators. We

820 recommend design-based inference (Särndal et al., 1992) as the framework within which

821 estimation is conducted. A fundamental tenet of design-based inference is that the specific 
822 estimators for accuracy, area, and the variances of these estimators depend on the sampling

823 design implemented; different estimators are appropriate for different sampling designs. It is,

824 Ttherefore, it is essential that only unbiased or consistent estimators should be used. In practical

825 terms, this means that only formulas for estimating parameters and variances that account for the

826 inclusion probabilities associated with the sampling design implemented should be used. All

827 recommended good practice estimators meet this condition, but the versions of the estimators

828 presented are usually forms where the individual inclusion probabilities do not appear explicitly.

\section{$829 \quad$ 4.3 Estimating Accuracy}

830 The cell entries of the error matrix and the population parameters derived from it must be

831 estimated from a sample. Suppose the sample-based estimator of $p_{i j}$ is denoted as $\hat{p}_{i j}$. Once $\hat{p}_{i j}$

832 is available for each element of the error matrix, parameters can be estimated by substituting $\hat{p}_{i j}$

833 for $p_{i j}$ in the formulas for the parameters. Accordingly, the error matrix should be reported in

834 terms of these estimated area proportions, $\hat{p}_{i j}$, and not in terms of sample counts, $n_{i j}$. The

835 specific formula for estimating $p_{i j}$ depends on the sampling design used. For equal probability

836 sampling designs (e.g., simple random and systematic sampling) and stratified random sampling

837 in which the strata correspond to the map classes,

838

$839 \hat{p}_{i j}=W_{i} \frac{n_{i j}}{n_{i}}$

841 where $W_{i}$ is the proportion of area mapped as class $i$. For simple random and systematic

842 sampling, Eq. (4) is a poststratified estimator of $p_{i j}$ (Card, 1982) and for these sampling designs

843 the poststratified estimator is recommended because it will have better precision than the 
844 estimators commonly used (cf. Stehman and Foody, 2009). Substituting $\hat{p}_{i j}$ of Eq. (4) into

845 Eqns. 1-3 yields estimators of overall, user's, and producer's accuracies. These formulas are

846 simpler special cases of a more general estimation approach described in Strahler et al. (2006,

847 Eqn. 3.1).

848 The sampling variability associated with the accuracy estimates should be quantified by

849 reporting standard errors. The variance estimators are provided below, and taking the square root

850 of the estimated variance results in the standard error of the estimator. For overall accuracy, the

851 estimated variance is

852

$853 \widehat{V}(\widehat{O})=\sum_{i=1}^{q} W_{i}^{2} \widehat{U}_{i}\left(1-\widehat{U}_{i}\right) /\left(n_{i \cdot}-1\right)$

854

855 For user's accuracy of map class $i$, the estimated variance is

856

857

$\widehat{V}\left(\widehat{U}_{i}\right)=\widehat{U}_{i}\left(1-\widehat{U}_{i}\right) /\left(n_{i}-1\right)$

858

859 For producer's accuracy of reference class $j=k$, the estimated variance is

860

$861 \widehat{V}\left(\widehat{P}_{j}\right)=\frac{1}{\widehat{N}_{\cdot j}^{2}}\left[\frac{N_{j .}^{2}\left(1-\hat{P}_{j}\right)^{2} \widehat{U}_{j}\left(1-\widehat{U}_{j}\right)}{n_{j .}-1}+\widehat{P}_{j}^{2} \sum_{i \neq j}^{q} N_{i \cdot}^{2} \frac{n_{i j}}{n_{i \cdot}}\left(1-\frac{n_{i j}}{n_{i \cdot}}\right) /\left(n_{i \cdot}-1\right)\right]$

862

863 where $\widehat{N}_{\cdot j}=\sum_{i=1}^{q} \frac{N_{i}}{n_{i} .} n_{i j}$ is the estimated marginal total number of pixels of reference class $j, N_{j}$.

864 is the marginal total of map class $j$ and $n_{j}$. is the total number of sample units in map class $j$.

865 These are the usual variance estimators applied to the stratified sampling, and the estimators 
would be viewed as poststratified variance estimators for simple random and systematic

867 sampling. For systematic sampling, the variance estimators are approximations that usually result

868 in overestimation of variance. These variance estimators are also based on assumptions that the

869 assessment unit for the response design is a pixel and each pixel has a hard classification for the

870 map and a hard classification for the reference data. The variance estimators would not apply to a

871 polygon assessment unit or to a mixed pixel situation.

\section{$872 \quad 4.4$ Estimating Area}

873 The error matrix also provides the basis for estimating the area of classes such as those

874 representing change and no-change. Indeed, $\underline{t}$ The population error matrix (Table 4) provides two

875 different approaches for estimating the proportion of area. Suppose we are interested in

876 estimating the proportion of area of class $k$. The row and column totals are the sums of the $p_{i j}$

877 values in the respective rows and columns. Thus, the row total $p_{k}$. represents the proportion of

878 area mapped as class $k$ (e.g., if $k$ is a change class such as forest loss then $p_{k}$. is the proportion of

879 area mapped as forest loss) and the column total $p_{\cdot k}$ represents the proportion of area of class $k$

880 as determined from the reference classification (e.g., $p_{\cdot k}$ would be the proportion of area of forest

881 loss as determined from the reference classification).

882 The two area proportion parameters for class $k$ (i.e., $p_{k}$. and $p_{\cdot k}$ ) are unlikely to have the

883 same value, so a decision arises as to which parameter should be the focus. Once a change map is

884 complete, $p_{k}$. is known, but because the reference classification is available only for a sample,

$885 p_{\cdot k}$ must be estimated from the sample. Consequently, the need to estimate $p_{\cdot k}$ introduces

886 uncertainty in the form of sampling variability, whereas $p_{k}$. is not subject to sampling variability

887 (Stehman, 2005).The map-based parameter $p_{k}$. is known with certainty but likely biased because

888 of classification error. Conversely, $p_{\cdot k}$ is determined from the reference classification ${ }_{\cdot-}$ and, 
889 therefore, $p_{\cdot k}$ should have smaller bias than $p_{k}$. (i.e., the bias attributable to reference data error 890 is smaller than the bias attributable to map classification error). The "good practice" guidelines

891 are founded on the premise that the reference classification is of superior quality to the map

892 classification and that the sampling design implemented yields estimates with small standard

893 errors. Consequently, we recommend that area estimation should be based on $p_{\cdot k}$, the proportion

894 of area derived from the reference classification.

895 A variety of estimators has been proposed for estimating $p_{\cdot k}$ from the error matrix. For any

896 sampling design and response design leading to an estimated error matrix with $p_{i j}$ in terms of

897 proportion of area, a direct estimator of the proportion of area of class $k$ is

898

$899 \hat{p}_{\cdot k}=\sum_{i=1}^{q} \hat{p}_{i k}$

900

901 This estimator is simply the sum of the estimated area proportions of class $k$ as determined from

902 the reference classification (i.e., the sum of column $k$ of the estimated error matrix). If the

903 sampling design is simple random, systematic, or stratified random with the map classes defined

904 as the strata, Eq. (8) would be computed using $\hat{p}_{i j}=W_{i} \frac{n_{i j}}{n_{i}}$ leading to the often used special

905 case estimator

906

$907 \hat{p}_{\cdot k}=\sum_{i=1}^{q} W_{i} \frac{n_{i k}}{n_{i}}$

908

909 This estimator is a poststratified estimator for simple random and systematic sampling, and it is

910 the direct stratified estimator of $p_{\cdot k}$ for stratified random sampling when the map classes are the 911 strata. For these sampling designs, the stratified estimator (Eq. 9) generally has better precision 
912 than a variety of alternative estimators of area (Stehman, 2013) and consequently the stratified

913 estimator is recommended.

914 For the stratified estimator of proportion of area (Eq. 9), the standard error is estimated by

915

$916 \quad S\left(\hat{p}_{\cdot k}\right)=\sqrt{\sum_{i} W_{i}^{2} \frac{\frac{n_{i k}}{n_{i \cdot}}\left(1-\frac{n_{i k}}{n_{i \cdot}}\right)}{n_{i \cdot}-1}}=\sqrt{\sum_{i} \frac{W_{i} \hat{p}_{i k}-\hat{p}_{i k}^{2}}{n_{i \cdot}-1}}$

918 where $n_{i k}$ is the sample count at cell $(i, k)$ in the error matrix, $W_{i}$ is the area proportion of map

919 class $i$, and the summation is over the $q$ classes. For systematic sampling, Eq. (10) is an

920 approximation that is typically an overestimate for the actual standard error of systematic

921 sampling. The estimated area of class $k$ is $\hat{A}_{k}=A \times \hat{p}_{\cdot k}$, where $A$ is the total map area. The

922 standard error of the estimated area is given by

923

$924 S\left(\hat{A}_{k}\right)=A \times S\left(\hat{p}_{\cdot k}\right)$

925

926 An approximate $95 \%$ confidence interval is obtained as $\hat{A}_{k} \pm 1.96 \times S\left(\hat{A}_{k}\right)$.

927 5. Example of Good Practices: Estimating Area and Assessing

928 Accuracy of Forest Change

929 The following hypothetical example illustrates the workflow of assessing accuracy of a forest

930 change map and estimating area. Consider a change map for 2000 to 2010 consisting of two

931 change classes and two stable classes: deforestation, forest gain, stable forest and stable non-

932 forest. The map was produced by supervised classification of data from Landsat ETM+ with the 
933 objective of estimating the gross rates of forest loss and gain. The first step in the assessment was

934 to visually inspect the change map and identify obvious errors by comparing the classified results

935 to the Landsat data of 2000 and 2010. Misclassified regions were relabelled before proceeding to

936 the rigorous evaluation of the map. After obvious errors were removed, the areas of the map

937 classes were 200,000 Landsat pixels (18,000 ha) of deforestation, 150,000 pixels (13,500 ha) of

938 forest gain, 3,200,000 pixels (288,000 ha) of stable forest, and 6,450,000 pixels (580,500 ha) of

939 stable non-forest. The two change classes thus occupy $3.5 \%$ of the total map area. The accuracy

940 assessment was designed for the objectives of estimating overall and class-specific accuracies,

941 areas of the individual classes (as determined by the reference classification), and confidence

942 intervals for each accuracy and area parameter. The spatial assessment unit in this example is a

943 Landsat pixel $(30 \mathrm{~m} \times 30 \mathrm{~m})$.

\section{$944 \quad 5.1$ Sampling Design}

945 A stratified random sampling design with the four map classes as strata adheres to the

946 recommended practices outlined in Section 2.3 and satisfies the accuracy assessment and area

947 estimation objectives. In the next two subsections, we present sample size and sample allocation

948 planning calculations for the stratified design. Sample size planning is an inexact science because

949 it is dependent on information on-accuracy and area information that must be speculative prior to

950 conducting the actual accuracy assessment. Nevertheless, these planning calculations can provide

951 informative insight into the choices of sample size and sample allocation to strata.

\section{5.1.1 Determining the Sample Size}

953 For simple random sampling and targeting overall accuracy as the estimation objective, Cochran

954 (1977, Eq. 4.2) suggests using a sample size of 
$956 n=\frac{z^{2} O(1-O)}{d^{2}}$

958 where $O$ is the overall accuracy expressed as a proportion, $z$ is a percentile from the standard 959 normal distribution $(z=1.96$ for a $95 \%$ confidence interval, $z=1.645$ for a $90 \%$ confidence

960 interval), and $d$ is the desired half-width of the confidence interval of $O$. Eq. (12) provides a

961 starting point for assessing sample size for the limited scope of estimating overall accuracy.

962 For stratified random sampling, Cochran (1977, Eq. 5.25) provides the following sample size

963 formula (the cost of sampling each stratum is assumed the same):

964

$965 \quad n=\frac{\left(\sum W_{i} S_{i}\right)^{2}}{[S(\hat{O})]^{2}+(1 / N) \sum W_{i} S_{i}^{2}} \approx\left(\frac{\sum W_{i} S_{i}}{S(\hat{O})}\right)^{2}$

966

967 where $N=$ number of units in the ROI, $S(\hat{O})$ is the standard error of the estimated overall

968 accuracy that we would like to achieve, $W_{i}$ is the mapped proportion of area of class $i$, and $S_{i}$ is

969 the standard deviation of stratum $i, S_{i}=\sqrt{U_{i}\left(1-U_{i}\right)}$ (Cochran, 1977, Eq. 5.55). Because $N$ is

970 typically large (e.g., over 10 million pixels in this example), the second term in the denominator

971 of Eq. (13) can be ignored. We specify a target standard error for overall accuracy of 0.01.

972 Suppose from past experience with similar change mapping efforts we know that errors of

973 commission are relatively common for the change classes while the stable classes are more

974 accurate (e.g., Olofsson et al., 2010; 2011). Consequently, we conjecture that user's accuracies of

975 the two change classes will be 0.70 for deforestation and 0.60 for forest gain, and user's

976 accuracies of the stable classes will be 0.90 for stable forest and 0.95 for stable non-forest. The 
977 resulting sample size from Eq. (13) is $n=641$. These sample size calculations should be repeated

978 for a variety of choices of $S(\hat{O})$ and $U_{i}$ before reaching a final decision.

980 Once we have chosen the overall sample size is chosen, we determine the allocation of the 981 sample to strataneeds be determined. It is important that the sample size allocation results in 982 precise estimates of accuracy and area. Stehman (2012) identifies four different approaches to 983 sample allocation: proportional, equal, optimal and power allocation. In proportional allocation, 984 the sample size per map class is proportional to the relative area of the map class. In this 985 example, and which is usually the case when mapping land change, the mapped areas of change 986 are small relative to other classes so proportional allocation will lead to small sample sizes in the 987 rare classes (unless $n$ is very large) and imprecise estimates of user's accuracy for these rare 988 classes. Allocating an equal sample size to all strata targets estimation of user's accuracy of each 989 map class but equal allocation is not optimized for estimating area and overall accuracy. Neyman 990 optimal allocation (Cochran, 1977) can be used to minimize the variance of the estimator of 991 overall accuracy or the estimator of area, but optimal allocation becomes difficult to implement 992 when multiple estimation objectives are of interest as will be the case when estimating accuracy 993 and area of several land-cover classes or land-cover change types.

994 We suggest the following simplified approach to sample size allocation. Allocate a sample 995 size of 50-100 for each change strata using the variance estimator for user's accuracy (Eq. 6) to 996 decide the sample size needed to achieve certain standard errors for the assumed estimated user's 997 accuracy for that class. The sample size allocated to these rare class strata will also be affected 998 by the total sample size, $n$, available to allocate. A small overall sample sizen might allow for 999 only 50 sample units per rare class stratum. Suppose that $n-r$ sample units remain after a sample 
1000 size of $r$ units has been allocated to the rare class strata. The sample size of $n-r$ is then allocated 1001 proportionally to the area of each remaining stratum. The anticipated estimated variances can 1002 then be computed (based on the sample size allocation) for user's and overall accuracy and area 1003 using Eqs. (5), (6) and (10). The sample size allocation process can be iterated until an allocation 1004 is found that yields satisfactory anticipated standard errors for the key accuracy and area 1005 estimates. The effect of the choice of sample allocation will be observed in the standard errors of 1006 the estimates, however, a poor allocation of sample size to strata will not result in biased 1007 estimators.

1008 In this example, we know the mapped areas of the four map classes $\left(W_{i}\right)$, we have 1009 conjectured values of user's accuracies and standard errors of the strata, and we have estimated a 1010 total sample size of 641 (Table 5). The resulting sample sizes for proportional and equal 1011 allocation are shown in Table 5. As described above, neither of these is optimal and we want to 1012 find a compromise between the two. We start by allocating 100 sample units each to the change 1013 classes and then allocate the remainder of the sample size proportionally to the stable classes.

1014 This gives the allocation in column "Alloc1". Since the recommendation is to allocate between 101550 and 100 sample units in the change strata, we introduce two additional allocations with 75 and 101650 sample units in the change strata, respectively (“Alloc2" and "Alloc3"). To determine which 1017 of these allocations to use, we need to examine the standard errors of the estimated user's 1018 accuracy, estimated overall accuracy, and estimated areas using Eq. (5), (6) and (10). 1019 1020 $<<$ TABLE 5 HERE $>>$ 
1022 It is necessary to speculate the outcome of the accuracy assessment to compute the anticipated

1023 standard errors for each sample allocation considered. The hypothesized error matrix in Table 6

1024 reflects the anticipated outcome that the change classes will be rare and have lower class-specific

1025 accuracies than the two stable classes. The population error matrix was also constructed to yield

1026 the hypothesized accuracies input into the sample size planning calculations of the previous

1027 section. When creating the hypothesized error matrix used for sample size and sample allocation

1028 planning, we should draw upon any past experience for insight into the accuracy of the map to be 1029 produced.

1033 Table 7 shows the standard errors of the user's and overall accuracies and estimated areas of both 1034 deforestation and stable forest for each of the five sample allocations in Table 5 and the

1035 hypothetical population error matrix of Table 6. No single allocation is best for all estimation 1036 objectives, so a choice among competing objectives is necessary. The emphasis on prioritizing 1037 objectives during the planning stage (Section 2) becomes particularly relevant to the decision of 1038 sample allocation because different allocations favour different estimation objectives. For 1039 example, equal allocation gives the smallest standard error of the user's accuracy of deforestation 1040 but a high standard error of the estimated area of deforestation. Proportional allocation will result 1041 in smaller standard errors of overall accuracy and area of stable forest but the standard error for 1042 estimated user's accuracy of deforestation is two to four times larger than the corresponding 1043 standard errors for other sample allocations. In this case, "Alloc1-3" provide allocations that 
1044 generate relatively small standard errors for the different estimates. We will choose "Alloc2" 1045 with 75 sample units in the two change classes.

\subsection{Estimating Accuracy, Area and Confidence Intervals}

1049 To create the reference classification for labelling each sample unit, a combination of Landsat 1050 data from the USGS open archive together with GoogleEarth ${ }^{\mathrm{TM}}$ provides a source of cost free 1051 reference data. Our hypothetical map was produced using Landsat, and the good practice

1052 recommendations stipulate that if using the same data for creation of both the map and reference 1053 classifications, the process of creating the latter should be of higher quality than the map-making

1054 process. The process of labelling the sample units thus has to be more accurate than supervised 1055 classification. A manual inspection by three analysts of each of the sample units using a set of 1056 Landsat images together with GoogleEarth ${ }^{\mathrm{TM}}$ imagery acquired around the same time as the 1057 images used to make the map is assumed to be a more accurate process than supervised 1058 classification. Suppose $t \underline{T}$ he error matrix resulting from this response design and sample is 1059 presented in terms of the sample counts displayed in Table 8, and the computations for the 1060 accuracy and area estimates are detailed in the following two subsections. $<<$ TABLE 8 HERE $>>$

\subsubsection{Estimating Accuracy}

1065 Because the sampling design is stratified random using the map classes as strata, the cell entries 1066 of the error matrix are estimated using Eq. (4). 
1070 We can now estimate user's accuracy $\widehat{U}_{i}=\frac{\hat{p}_{i i}}{\hat{p}_{i}}$; producer's accuracy $\widehat{P}_{j}=\frac{\hat{p}_{j j}}{\hat{p}_{\cdot j}}$; and overall 1071 accuracy $\hat{O}=\sum_{j=1}^{q} \hat{p}_{j j}$ using the estimated area proportions. Variances for these accuracy 1072 measures are estimated using Eq. (5)-(7). 95\% confidence intervals are estimated as

$1073 \pm 1.96 \sqrt{\widehat{V}\left(\widehat{U}_{i}\right)}$ (replace $\widehat{U}_{i}$ with $\widehat{P}_{j}$ and $\hat{O}$ for the producer's and overall accuracies). In this case, 1074 the estimated user's accuracy ( $\pm 95 \%$ confidence interval) is $0.88 \pm 0.07$ for deforestation, $10750.73 \pm 0.10$ for forest gain, $0.93 \pm 0.04$ for stable forest, and $0.96 \pm 0.02$ for stable non-forest. 1076 The estimated producer's accuracy is $0.75 \pm 0.21$ for deforestation, $0.85 \pm 0.23$ for forest gain, $10770.93 \pm 0.03$ for stable forest, and $0.96 \pm 0.01$ for stable non-forest. The estimated overall 1078 accuracy is $0.95 \pm 0.02$.

\section{5.2.2. Estimating Area and Uncertainty}

1080 The next step is to use the estimated area proportions in Table 9 to estimate the area of each 1081 class. The row totals of the error matrix in Table 9 give the mapped area proportions (which are 1082 also given by $W_{i}$ ) while the column totals give the estimated area proportions according to the 1083 reference data. Multiplying the latter by the total map area gives the stratified area estimate of 1084 each class according to the reference data. For example, the estimated area of deforestation 1085 according to the reference data is $\hat{A}_{1}=\hat{p}_{\cdot 1} \times A_{\text {tot }}=0.024 \times 10,000,000$ pixels $=235,086$ 1086 pixels $=21,158$ ha. The mapped area of deforestation $\left(A_{m, 1}\right)$ of 200,000 pixels was thus 1087 underestimated by 35,086 pixels or 3,158 ha. 
1088 The second step is to estimate a confidence interval for the area of each class. From Eq. (10), $1089 S\left(\hat{p}_{\cdot 1}\right)=0.0035$ and the standard error for the estimated area of forest loss is $S\left(\hat{A}_{1}\right)=S\left(\hat{p}_{\cdot 1}\right) \times$

$1090 A_{t o t}=0.0035 \times 10,000,000=34,097$ pixels. The margin of error of the confidence interval

1091 is $1.96 \times 34,097=68,418$ pixels $=6,158$ ha. We have thus estimated the area of deforestation

1092 with a $95 \%$ confidence interval: $21,158 \pm 6,158$ ha. The area estimate with a $95 \%$ confidence

1093 interval of the forest gain class is $11,686 \pm 3,756$ ha; stable forest is $285,770 \pm 15,510$ ha and

1094 stable non-forest 581,386 $\pm 16,282$ ha.

1095 This example has illustrated the workflow of assessing accuracy, and estimating area and 1096 confidence intervals of area of the classes of a change map. While this is fairly straightforward

1097 once the error matrix has been constructed, the example highlights the need to consider different 1098 objectives when designing the sample.

1099 A tool for estimating unbiased accuracy measures and areas with $95 \%$ confidence intervals 1100 can be downloaded from www.people.bu.edu/olofsson/ (click 'Research'> 1101 'Accuracy/Uncertainty'). The tool is implemented in MatlabT.

\section{6. Summary}

1103 Conducting an accuracy assessment of a land change map serves multiple purposes. In addition 1104 to the obvious purpose of quantifying the accuracy of the map, the reference sample serves as the

1105 basis of estimates of area of each class where area is defined by the reference classification, and

$1106 \notin$ The accuracy assessment sample data also contribute to estimates of uncertainty of the area

1107 estimates. Without an accuracy assessment, there is no way to communicate map quality in a 1108 quantitative and meaningful fashion. We acknowledge that there is no singular "best" approach 1109 and the recommendations provided do not preclude the existence of other acceptable practices. 
1110 However, by following the "good practice" recommendations presented by this paper, scientific

1111 credibility of the accuracy and area estimates is ensured. The "good practice" recommendations

1112 are summarized as follows, organized by the three major components of the accuracy assessment

1113 methodology, the sampling design, response design, and analysis:

$1114 \quad 6.1$ General

1115 - Visually inspect the map and correct obvious errors before conducting the accuracy

$1116 \quad$ assessment

1117 - Accuracy and area estimates will be determined from a classification (i.e., the reference

1118 classification) that is of higher quality than the land change map being evaluated

1119 - A sampling approach is needed because the cost of obtaining the reference classification

1120 for the entire region of interest will be prohibitive

1121 - The sample used for accuracy assessment and area estimation is separate from

1122 (independent of) the data used to train or develop the classification

\section{$1123 \quad 6.2$ Sampling design}

1124 - Implement a probability sampling design to provide a rigorous foundation via design-

1125 based sampling inference

1126 - Document and quantify any deviations from the probability sampling protocol

1127 - Choose a sampling design on the basis of specified accuracy objectives and prioritized

1128 desirable design criteria

1129 - Sampling design guidelines

$1130 \quad 0$ Stratify by map class to reduce standard errors of class-specific accuracy

$1131 \quad$ estimates 
- If resources are adequate, stratify by subregions to reduce standard errors of subregion-specific estimates

- Use cluster sampling if it provides a substantial cost savings or if the objectives require a cluster unit for the assessment

- Both simple random and systemic selection protocols are acceptable options

- The recommended allocation of sample size to strata (assuming the map classes are the strata) is to increase the sample size for rare change classes to achieve an acceptable standard error for estimated user's accuracies and to allocate the remaining sample size roughly proportional to the area occupied by the common classes

- Use sample size and optimal allocation planning calculations as a guide to decisions on total sample size and sample allocation

- Evaluate the potential outcome of sample size and sample allocation decisions on the standard errors of accuracy and area estimates for hypothetical error matrices based on the anticipated accuracy of the map

- Stratified random sampling using the map classification to define strata is a simple, but generally applicable design that will typically satisfy most accuracy and area estimation objectives and desirable design criteria

\subsection{Response design}

- Reference data should be of higher quality than the data used for creating the map, or if using the same source, the process of creating the reference classification should be more accurate than the process of creating the map

- High overhead cost may eliminate field visits as a source of reference data 
1154 - The reference data should provide sufficient temporal representation consistent with the 1155 change period of the map

1156 - Data from the Landsat open archive in combination with high spatial resolution imagery 1157 provide a low-cost and often useful source of reference data (national photograph

1158 archives, satellite photo archives (e.g., Kompsat), and the collections available through

1159 Google Earth ${ }^{\mathrm{TM}}$ are possible high resolution imagery sources)

1160 - Specify protocols for accounting for uncertainty in assigning the reference classifications

1 - Assign each sample unit a primary and secondary label (secondary not required if there is 1162 highly confidencet in the primary label)

1163 - Include an interpreter specified confidence for each reference label (e.g., high, medium, $1164 \quad$ or low confidence)

1165 Implement protocols to ensure consistency among individual interpreters or teams of 1166 interpreters

1167 Specify a protocol for defining agreement between the map and reference classifications 1168 that will lead to an error matrix expressed in terms of proportion of area

\section{$1169 \quad 6.4$ Analysis}

1170 - Report the error matrix in terms of estimated area proportions

1171 - Report the area (or proportion of area) of each class as determined from the map

1172 - Report user's accuracy (or commission error), producer's accuracy (or omission

1173 error), and overall accuracy (Equations 1-3)

1174 Avoid use of the kappa coefficient of agreement for reporting accuracy of land $1175 \quad$ change maps 
- Estimate the area of each class according to the classification determined from the reference data

- Use estimators of accuracy and area that are unbiased or consistent

- For simple random, systematic, and stratified random sampling when the map classes are defined as strata, use stratified estimators of accuracy (Eqs. 5-7) and a stratified estimator of area (Eq. 9)

- Quantify sampling variability of the accuracy and area estimates by reporting standard errors or confidence intervals

- Use design-based inference to define estimator properties and to quantify uncertainty

- Assess the impact of reference data uncertainty on the accuracy and area estimates

1186 The recommendations provided are intended to serve as guidelines for choosing from among 1187 options of sampling design, response design, and analysis that will yield rigorous and defensible 1188 accuracy and area estimates. But good practice is not static. As improvements in technology

1189 become available and new methods are developed, good practice recommendations will evolve 1190 over time. Also, as practical experience accumulates with using new technology and 1 191 methodologiesy, good practice recommendations will be further amended to provide even more 1192 efficient yet still rigorous methods to estimate accuracy and area of land change. 


\section{References}

1195 Achard, F., Eva, H., Stibig, H.-J., Mayaux, P., Gallego, J., Richards, T., and Malingreau, J.-P.

1196 (2002). Determination of deforestation rates of the world's humid tropical forests. Science, $1197 \quad 297,999-1002$.

1198 Ahlqvist, O. (2008). In search of classification that supports the dynamics of science: The FAO

1199 Land Cover Classification System and proposed modifications. Environment and Planning B:

1200 Planning and Design, 35, 169-186.

1201 Baker, B. A., Warner, T. A., Conley, J. F., and McNeil, B. E. (2013). Does spatial resolution

1202 matter? A multi-scale comparison of object-based and pixel-based methods for detecting

1203 change associated with gas well drilling operations. International Journal of Remote Sensing, $1204 \quad 34,1633-1651$.

1205 Binaghi, E., Brivio, P. A., Ghezzi, P., and Rampini, A. (1999). A fuzzy set-based accuracy

1206 assessment of soft classification. Pattern Recognition Letters, 20, 935-948.

1207 Cakir, H. I., Khorram, S., and Nelson, S. A. C. (2006). Correspondence analysis for detecting

1208 land cover change. Remote Sensing of Environment, 102, 306-317.

1209 Card, D. H. (1982). Using map category marginal frequencies to improve estimates of thematic

1210 map accuracy. Photogrammetric Engineering and Remote Sensing, 49, 431-439

1211 Cochran, W. G. (1977). Sampling Techniques ( $3^{\text {rd }}$ ed.). New York: John Wiley \& Sons

1212 Cohen, W. B., Yang, Z., and Kennedy, R. (2010). Detecting trends in forest disturbance and

1213 recovery using yearly Landsat time series: 2. TimeSync - Tools for calibration and

1214 validation. Remote Sensing of Environment, 114, 2911-2924. 
1215 Comber A. J., Wadsworth, R. A., and Fisher, P. F. (2008). Using semantics to clarify the 1216 conceptual confusion between land cover and land use: the example of 'forest'. Journal of $1217 \quad$ Land Use Science, 3, 185-198.

1218 Congalton, R., and Green, K. (2009). Assessing the Accuracy of Remotely Sensed Data:

1219 Principles and Practices ( $2^{\text {nd }}$ ed.). Boca Raton: CRC/Taylor \& Francis

1220 DeFries, R., Houghton, R. A., Hansen, M., Field, C., Skole, D. L., and Townshend, J. (2002).

1221 Carbon emissions from tropical deforestation and regrowth based on satellite observations 1222 for the 1980s and 90s. Proceedings of the National Academy of Sciences, 99, 14256-14261.

1223 DeFries, R., Achard, F., Brown, S., Herold, M., Murdiyarso, D., Schlamadinger, B., and Souza, 1224 C. M. (2007). Earth observations for estimating greenhouse gas emissions from deforestation 1225 in developing countries. Environmental Science and Policy, 10, 385-394.

1226 de Sy, V., Herold, M., Achard, F., Asner, G. P., Held, A., Kellndorfer, J., and Verbesselt, J.

1227 (2012). Synergies of multiple remote sensing data sources for REDD+ monitoring. Current 1228 Opinion in Environmental Sustainability, 4, 696-706.

1229 Drummond, M. A., and Loveland T. R. (2010). Land-use pressure and a transition to forest-cover $1230 \quad$ loss in the eastern United States. BioScience, 60, 286-298.

1231 Duro, D. C., Franklin, S. E., and Duba, M. G. (2012). A comparison of pixel-based and object1232 based image analysis with selected machine learning algorithms for the classification of 1233 agricultural landscapes using SPOT-5 HRG imagery. Remote Sensing of Environment, $1234 \quad 118: 259-272$.

1235 Falkowski, M. J., Wulder, M. A., White, J. C., and Gillis, M. D. (2009). Supporting large-area, 1236 sample-based forest inventories with very high spatial resolution satellite imagery. Progress 1237 in Physical Geography, 33, 403-423. 
1238 FAO (2010). Global Forest Resources Assessment 2010. Food and Agriculture Organization of 1239 the United Nations.

1240 FAO (2011). Food and Agriculture Organization of the United Nations. Assessing forest 1241 degradation. Towards the development of globally applicable guidelines. Forest Resources 1242 Assessment Working Paper 177.

1243 Foody, G. M. (1992). On the compensation for chance agreement in image classification 1244 accuracy assessment. Photogrammetric Engineering and Remote Sensing, 58, 1459-1460

1245 Foody, G. M., Campbell, N. A., Trodd, N. M., and Wood, T. F. (1992). Derivation and 1246 applications of probabilistic measures of class membership from the maximum likelihood 1247 classification. Photogrammetric Engineering and Remote Sensing, 58, 1335-1341.

1248 Foody, G. M. (1996). Approaches for the production and evaluation of fuzzy land cover 1249 classifications from remotely sensed data. International Journal of Remote Sensing, 17, $1250 \quad 1317-1340$.

1251 Foody, G. M. (2002). Status of land cover classification accuracy assessment. Remote Sensing of 1252 Environment, 80, 185-201.

1253 Foody, G. M. (2010). Assessing the accuracy of land cover change with imperfect ground 1254 reference data. Remote Sensing of Environment, 114, 2271-2285.

1255 Foody, G. M., and Boyd, D. S. (2013). Using volunteered data in land cover map validation: 1256 mapping West African forests. IEEE Journal of Selected Topics in Applied Earth 1257 Observation and Remote Sensing, in press. DOI: 10.1109/JSTARS.2013.2250257 1258 Foody, G. M., See, L., Fritz, S., Van der Velde, M., Perger, C., Schill, C., and Boyd, D. S.
1259 (2013). Assessing the accuracy of volunteered geographic information arising from multiple
1260 centributors to an internet based collaborative project. Transactions in GIS, in press. 
1261 Foody, G. M. (2013). Ground reference data error and the mis-estimation of the area of land 1262 cover change as a function of its abundance. Remote Sensing Letters, in press.

1263 Gallego, F. J. (2012). The efficiency of sampling very high resolution images for area estimation 1264 in the European Union. International Journal of Remote Sensing, 33, 1868-1880.

1265 GOFC-GOLD (2011). A sourcebook of methods and procedures for monitoring and reporting 1266 anthropogenic greenhouse gas emissions and removals caused by deforestation, gains and 1267 losses of carbon stocks in forests remaining forests, and forestation. GOFC-GOLD Report 1268 version COP17-1, (GOFC-GOLD Project Office, Natural Resources Canada, Alberta, 1269 Canada).

1270 Gómez, C., White, J. C., and Wulder, M. A. (2011). Characterizing the state and processes of 1271 change in a dynamic forest environment using hierarchical spatio-temporal segmentation. 1272 Remote Sensing of Environment, 115, 1665-1679.

1273 Gopal, S., and Woodcock, C. (1994). Theory and methods for accuracy assessment of thematic 1274 maps using fuzzy sets. Photogrammetric Engineering and Remote Sensing, 60, 181-188.

1275 Grassi, G., Monni, S., Federici, S., Achard, F., and Mollicone, D. (2008) Applying the 1276 conservativeness principle to REDD to deal with the uncertainties of the estimates. $1277 \quad$ Environmental Research Letters, 3, 3.

1278 Hansen, M. C., Stehman, S. V., and Potapov, P. V. (2010). Quantification of global gross forest 1279 cover loss. Proceedings of the National Academy of Sciences, 107, 8650-8655.

1280 He, Y. H., Franklin, S. E., Guo, X. L., and Stenhouse, G. B. (2011). Object-orientated 1281 classification of multi-resolution images for the extraction of narrow linear forest 1282 disturbance. Remote Sensing Letters, 2, 147-155. 
1283 Herold, M., and Skutsch, M. (2011). Monitoring, reporting and verification for national REDD + 1284 programmes: two proposals. Environmental Research Letters 6014002.

1285 Herold, M., Román-Cuesta, R.M., Mollicone, D., Hirata, Y., Van Laake, P., Asner, G.P., Souza,

1286 C., Skutsch, M., Avitabile, V., and Macdicken, K. (2011). Options for monitoring and 1287 estimating historical carbon emissions from forest degradation in the context of REDD+. $1288 \quad$ Carbon balance and management, 6, 13

1289 Huang, C., Goward, S. N., Masek, J. G., Thomas, N., Zhu, Z., and Vogelmann, J. E. (2010). An 1290 automated approach for reconstructing recent forest disturbance history using dense Landsat 1291 time series stacks. Remote Sensing of Environment, 114, 183-198.

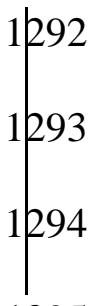

1295

1296

1297

1298

1299

1300

1301

1302

1303 1304

Нууррӓ, J., Нуурра, H., Inkinen, M., Engdahl, M., Linke, S., and Zhu, Y. H. (2000). Accuracy cemparison of various remote sensing data sources in the retrieval of forest stand attributes. Forest Ecology and Mangement, $128,109-120$.

Iwao, K., Nishida, K., Kinoshita, T., and Yamagata, Y. (2006). Validating land cover maps with Degree Confluence Project information. Geophysical Research Letters 33: L23404

Jeon, S. B., Olofsson, P., and Woodcock, C. E. (2013). Land use change in New England: a reversal of the forest transition. Journal of Land Use Science DOI: 10.1080/1747423X.2012.754962

Johnson, B. A. (2013). High-resolution urban land-cover classification using a competitive multi-scale object-based approach. Remote Sensing Letters, 4, 131-140.

Kelly, M., Estes, J. E., and Knight, K. A. (1999). Image interpretation keys for validation of global land-cover data sets. Photogrammetric Engineering \& Remote Sensing, 65, 10411050. 
1305 Kennedy, R., Yang, Z., and Cohen, W. B. (2010). Detecting trends in forest disturbance and 1306 recovery using yearly Landsat time series: 1 . LandTrendr - Temporal segmentation 1307 algorithms. Remote Sensing of Environment, 114, 2897-2910.

1308 Knight, J. F., and Lunetta R. S. (2003). An experimental assessment of minimum mapping unit 1309 size. IEEE Transactions on Geoscience and Remote Sensing, 40, 2132-2134.

1310 Kurz, W. A. (2010). An ecosystem context for global gross forest cover loss estimates.

$1311 \quad$ Proceedings of the National Academy of Science, 107, 9025-9026.

1312 Lewis, H. G., and Brown, M. (2001). A generalized confusion matrix for assessing area

1313 estimates from remotely sensed data. International Journal of Remote Sensing, 22, 3223-

13143235.

1315 Liu, C., Frazier, P., and Kumar, L., 2007. Comparative assessment of the measures of thematic 1316 classification accuracy. Remote Sensing of Environment, 107, 606-616.

1317 Lindberg, E., Olofsson, K., Holmgren, J., and Olsson, H. (2012). Estimation of 3D vegetation 1318 structure from waveform and discrete return airborne laser scanning data. Remote Sensing of 1319 Environment, 118, 151-161.

1320 Mayaux, P., Eva, H., Gallego, J., Strahler, A. H., Herold, M., Agrawal, S., Naumov, S., De

1321 Miranda, E. E., Di Bella, C. M., Ordoyne, C., Kopin, Y., and Roy, P. S. (2006). Validation of 1322 the Global Land Cover 2000 map. IEEE Transactions on Geoscience and Remote Sensing, $1323 \quad 44,1728-1739$.

1324 McRoberts, R. E., (2011). Satellite image-based maps: Scientific inference or pretty pictures? $1325 \quad$ Remote Sensing of Environment, 115, 715-724. 
1326 Olofsson, P., Torchinava, P., Woodcock, C. E., Baccini, A., Houghton, R. A., Ozdogan, M., 1327 Zhao, F., and Yang, X. (2010). Implications of Land Use Change on the National Terrestrial 1328 Carbon Budget of Georgia. Carbon Balance and Management, 5, 4.

1329 Olofsson, P., Kuemmerle, T., Griffiths, P., Knorn, J., Baccini, A., Gancz, V., Blujdea, V., 1330 Houghton, R.A., Abrudan, I.V., and Woodcock C.E. (2011). Carbon implications of forest 1331 restitution in post-socialist Romania, Environmental Research Letters, 6, 045202.

1332 Olofsson, P., Stehman, S. V., Woodcock, C. E., Sulla-Menashe, D., Sibley, A. M., Newell, J. D., 1333 Friedl, M. A., and Herold, M. (2012). A global land cover validation dataset, I: Fundamental 1334 design principles. International Journal of Remote Sensing, 33, 5768-5788

1335 Olofsson, P., Foody, G.M., Stehman, S. V., and Woodcock, C.E. (2013). Making better use of 1336 accuracy data in land change studies: estimating accuracy and area and quantifying 1337 uncertainty using stratified estimation. Remote Sensing of Environment, 129, 122-131

1338 Pontius, R. G. (2000). Quantification error versus location error in comparison of categorical 1339 maps. Photogrammetric Engineering \& Remote Sensing, 66, 1011-1016.

1340 Pontius, R. G., and Lippitt, C. D. (2006). Can Error Explain Map Differences Over Time?

1341 Cartography and Geographic Information Science, 33, 159-171.

1342 Pontius, R. G., and Millones, M. (2011). Death to kappa: birth of quantity disagreement and 1343 allocation disagreement for accuracy assessment. International Journal of Remote Sensing, $1344 \quad 32,4407-4429$.

1345 Powell, R., Matzke, N., de Souza, C., Clark, M., Numata, I., Hess, L., and Roberts, D. (2004). 1346 Sources of error in accuracy assessment of thematic land-cover maps in the Brazilian 1347 Amazon. Remote Sensing of Environment, 90, 221-234. 
1348 Pratihast, A. K., Herold, M., de Sy, V., Murdiyarso, D., and Skutsch, M. (2013). Linking 1349 community-based and national REDD+ monitoring: a review of the potential. Carbon $1350 \quad$ Management, 4, 91-104

1351 Riemann, R., Wilson, B. T., Lister, A., and Parks, S. (2010). An effective assessment protocol 1352 for continuous geospatial datasets of forest characteristics using USFS Forest Inventory and 1353 Analysis (FIA) data. Remote Sensing of Environment, 114, 2337-2352.

1354 Romijn, J. E., Herold, M., Kooistra, L., Murdiyarso, D., and Verchot, L. (2012). Assessing 1355 capacities of non-Annex I countries for national forest monitoring in the context of REDD+. 1356 Environmental Science and Policy, 20, 33-48.

1357 Sanz-Sanchez, M., Herold, M., and Penman, J. (2013). REDD+ related forest monitoring 1358 remains key issue: a report following the recent UN climate convention in Doha. Carbon 1359 Management, 4, 125-127.

1360 Särndal, C., Swensson, B., and Wretman, J. (1992). Model assisted survey sampling. New York: $1361 \quad$ Springer.

1362 Saura, S. (2002). Effects of minimum mapping unit on land cover data spatial configuration and 1363 composition. International Journal of Remote Sensing, 23, 4853-4880.

1364 Scepan, J. (1999). Thematic validation of high-resolution global land-cover data sets.

1365 Photogrammetric Engineering \& Remote Sensing, 65, 1051-1060.

1366 Schroeder, T. A., Wulder, M. A., Healey, S. P., and Moisen, G. G. (2011). Mapping wildfire and 1367 clearcut harvest disturbances in boreal forests with Landsat time series data. Remote Sensing 1368 of Environment, 115, 1421-1433.

1369 Skirvin, S. M., Kepner, W. G., Marsh, S. E., Drake, S. E., and Maingi, J. K., Edmonds, C. M., 1370 Watts, C.J., and Williams D. R. (2004). Assessing the accuracy of satellite-derived land- 
cover classification using historical aerial photography, digital orthophoto quadrangles, and airborne video data. In R. S. Lunetta and J. G. Lyon (Eds.), Remote Sensing and GIS Accuracy Assessment. Boca Raton: CRC Press.

1374 Stehman, S. V. (1997). Selecting and interpreting measures of thematic classification accuracy. 1375 Remote Sensing of Environment, 62, 77-89.

1376 Stehman, S. V. (2000). Practical implications of design-based sampling inference for thematic 1377 map accuracy assessment. Remote Sensing of Environment, 72, 35-45.

1378 Stehman S. V. (2001). Statistical rigor and practical utility in thematic map accuracy.

1379 Photogrammetric Engineering and Remote Sensing, 67, 727-734.

1380 Stehman, S. V. (2005). Comparing estimators of gross change derived from complete coverage 1381 mapping versus statistical sampling of remotely sensed data. Remote Sensing of 1382 Environment, 96, 466-474.

1383 Stehman, S. V. (2009). Sampling designs for accuracy assessment of land cover. International 1384 Journal of Remote Sensing, 30, 5243-5272.

1385 Stehman, S. V. (2012). Impact of sample size allocation when using stratified random sampling 1386 to estimate accuracy and area of land-cover change. Remote Sensing Letters, 3, 111-120.

1387 Stehman, S. V. (2013). Estimating area from an accuracy assessment error matrix. Remote 1388 Sensing of Environment, 132, 202-211.

1389 Stehman, S. V., and Czaplewski, R. L. (1998). Design and analysis for thematic map accuracy 1390 assessment: Fundamental principles. Remote Sensing of Environment, 64, 331-344.

1391 Stehman, S.V., and Foody, G.M. (2009). Accuracy Assessment. In T. A. Warner, M. D. Nellis, 1392 and G. M. Foody (Eds.) The SAGE Handbook of Remote Sensing. London: Sage $1393 \quad$ Publications. 
1394 Stehman, S. V., and Selkowitz, D. J. (2010). A spatially stratified, multi-stage cluster sampling 1395 design for assessing accuracy of the Alaska (USA) National Land-Cover Data (NLCD). 1396 International Journal of Remote Sensing, 31, 1877-1896

1397 Stehman, S. V., and Wickham, J. D. (2011). Pixels, blocks of pixels, and polygons: Choosing a 1398 spatial unit for thematic accuracy assessment. Remote Sensing of Environment. 115, 304413993055.

1400 Stehman, S. V., Sohl, T. L., and Loveland, T. R. (2003). Statistical sampling to characterize 1401 recent United States land-cover change. Remote Sensing of Environment, 86, 517-529.

1402 Stehman, S. V., Olofsson, P., Woodcock, C. E., Herold, M. and Friedl, M. A. (2012). A global 1403 land cover validation dataset, II: Augmenting a stratified sampling design to estimate 1404 accuracy by region and land-cover class. International Journal of Remote Sensing, 33:69756993

1406 Stehman, S. V., Wickham, J. D., Wade, T. G., and Smith, J. H. (2008). Designing a multi1407 objective, multi-support accuracy assessment of the 2001 National Land Cover Data (NLCD 1408 2001) of the conterminous United States. Photogrammetric Engineering \& Remote Sensing, $1409 \quad 74: 1561-1571$.

1410 Strahler, A. H., Boschetti, L., Foody, G. M., Friedl, M. A., Hansen, M. C., Herold, M., Mayaux, 1411 P., Morisette, J. T., Stehman, S. V., and Woodcock, C. E. (2006). Global land cover 1412 validation: Recommendations for evaluation and accuracy assessment of global land cover 1413 maps. EUR 22156 EN - DG, Office for Official Publications of the European Communities, $1414 \quad$ Luxembourg, 48 pp.

1415 Tomppo, E. O., Gschwantner, T., Lawrence, M., and McRoberts, R. E. (2010). National Forest 1416 Inventories: Pathways for Common Reporting, Springer, New York 
1417 UN-REDD (2008). UN Collaborative Programme on Reducing Emissions from Deforestation 1418 and Forest Degradation in Developing Countries (UN-REDD). FAO, UNDP, UNEP

1419 Framework Document.

1420 Wickham, J. D., Stehman, S.V., Fry, J.A., Smith, J.H., and Homer, C.G. (2001). Thematic

1421 accuracy of the NLCD 2001 land cover for the conterminous United States. Remote Sensing 1422 of the Environment, 114, 1286-1296.

1423 Wickham, J. D., Stehman, S. V., Gass, L., Dewitz, J., Fry, J. A., and Wade, T. G. (2013).

1424 Accuracy assessment of NLCD 2006 land cover and impervious surface. Remote Sensing of $1425 \quad$ Environment, 130, 294-304.

1426 Woodcock, C.E., Allen, R., Anderson, M., Belward, A., Bindschadler, R., Cohen, W., Gao, F., 1427 Goward, S.N., Helder, D., Helmer, E., Nemani, R., Oreopoulos, L., Schott, J., Thenkabail, 1428 P.S., Vermote, E.F., Vogelmann, J., Wulder, M.A., and Wynne, R. (2008). Free access to $1429 \quad$ Landsat imagery. Science, 320, 1011

1430 Wulder, M. A., Franklin, S., White, J. C., Linke, J., and Magnussen, S. (2006a). An accuracy 1431 assessment framework for large-area land cover classification products derived from medium 1432 resolution satellite data. International Journal of Remote Sensing, 27, 663-683.

1433 Wulder, M. A., White, J. C., Luther, J. E., Strickland, L. G., Remmel, T. K., and Mitchell, S. W. 1434 (2006b). Use of vector polygons for the accuracy assessment of pixel-based land cover maps. 1435 Canadian Journal of Remote Sensing, 32, 268-279.

1436 Wulder, M. A., White, J. C., Magnussen, S., and McDonald, S. (2007). Validation of a large area 1437 land cover product using purpose-acquired airborne video. Remote Sensing of Environment, $1438 \quad 106,480-491$. 
1439 1440

1441

1442

1443

1444

1445

1446

1447

1448

1449

1450

1451

1452

1453

Wulder, M. A., White, J. C., Hay, G. J., and Castilla, G. (2008a). Towards automated segmentation of forest inventory polygons on high spatial resolution satellite imagery. The Forestry Chronicle, 84, 221-230.

Wulder, M. A.; White, J. C.; Coops, N. C., and Butson, C. R. (2008b). Multi-temporal analysis of high spatial resolution imagery for disturbance monitoring. Remote Sensing of Environment. 112, 2729-2740.

Wulder, M. A., Masek, J. G., Cohen, W. B., Loveland, T. R., and Woodcock, C.E. (2012). Opening the archive: How free data has enabled the science and monitoring promise of Landsat. Remote Sensing of Environment, 122, 2-10.

Zimmerman, P.L., Housman, I.W., Perry, C.H., Chastain, R.A., Webb, J.B., and Finco, M.V. (2013). An accuracy assessment of forest disturbance mapping in the western Great Lakes. Remote Sensing of Environment, 128, 176-185

$\underline{\text { Table 1. Possible reference data sources }}$

Reference data source Exemplar citation

Field plots $\quad$ Hyyppä et al. 2000

Air photography $\quad \underline{\text { Skirvin et al. (2004) }}$

Forest inventory data $\quad \underline{\text { McRoberts (2011); Wulder et al. (2006b) }}$

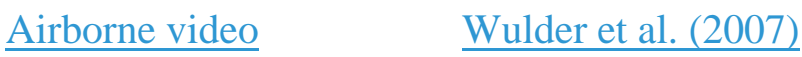

$\underline{\text { Lidar }} \quad \underline{\text { Lindberg et al. (2012) }}$

Satellite imagery $\quad \underline{\text { Scepan (1999); Cohen et al. (2010) }}$

Crowdsourcing $\quad$ Iwao et al. (2006); Foody and Boyd (2013) 
1454

1455 
$\begin{array}{ll}1456 & \text { Table 2. Example characteristics to record for each change polygon. Some attributes can be } \\ 1457 & \text { generated in the GIS; others will need to be entered by the analyst. Notion is that information is } \\ 458 & \text { captured and carried to provide insights and a record regarding the changes captured. The aim is } \\ 459 & \text { that the change polygons can be used in a manner that is invariant to source, but that metadata is } \\ 460 & \text { captured to explain or better understand any data related anomalies that may emerge. }\end{array}$ Attribute $\quad$ Definition/comments.

Change Area Area changed, e.g., polygon size in hectares

Change Perimeter Perimeter of polygon, in meters

Change Type Notation of change type, harvest, fire, insect, urban expansion, agricultural development

Change Date As possible, note the change date. May be available from other records, e.g., when a fire occurred, or the acquisition date of the image or photography used.

Data Source $\quad$ Note the data source from which the change polygon is made

Analyst $\quad$ Name or code to denote the interpreter

Date Interpreted Note the date when the interpretation occurred 
Table 3. Elements for consideration when selecting reference data

\begin{tabular}{|c|c|}
\hline Element & Considerations \\
\hline$\underline{\text { Cost }}$ & $\begin{array}{l}\text { What is the budget? What amount per unit of reference data can be } \\
\text { purchased? Is the interpretation / labelling protocol efficient? }\end{array}$ \\
\hline Ease of access & $\begin{array}{l}\text { Varies by data type. Can field visits be made? Is archival image data } \\
\text { available? }\end{array}$ \\
\hline Ease of use & $\begin{array}{l}\text { Is the data produced in a consistent fashion? Is it in formats that are } \\
\text { commonly used? }\end{array}$ \\
\hline Opportunity for & Can protocols be developed and applied in a systematic and repetitive \\
\hline$\underline{\text { consistency }}$ & fashion? Can some tasks be automated? \\
\hline $\begin{array}{l}\text { Vintage - temporal } \\
\text { representation }\end{array}$ & $\begin{array}{l}\text { Is the data representative of a time or time period that is relevant to the } \\
\text { change product under consideration? }\end{array}$ \\
\hline Spatial coverage & $\begin{array}{l}\text { Are there opportunities for multiple reference sites from a given } \\
\text { reference data source? }\end{array}$ \\
\hline Interpretability of & Does the data source capture and portray the change types of interest? \\
\hline change types & E.g., is the spatial resolution sufficiently fine to enable interpretation? \\
\hline Geolocation & Can the candidate reference data source be assumed to be accurately \\
\hline
\end{tabular}




\begin{tabular}{|c|c|c|c|c|c|c|}
\hline \multicolumn{7}{|c|}{ proportion of area as suggested by good practice recommendations. } \\
\hline \multicolumn{7}{|c|}{$\underline{\text { Reference }}$} \\
\hline \multirow{6}{*}{ 워 } & _ & Class 1 & Class 2 & Class 3 & Class 4 & Total \\
\hline & $\underline{\text { Class } 1}$ & $\underline{p_{11}}$ & $p_{12}$ & $p_{13}$ & $p_{14}$ & $p_{1}$. \\
\hline & $\underline{\text { Class } 2}$ & $p_{21}$ & $p_{22}$ & $p_{23}$ & $p_{24}$ & $p_{2}$ \\
\hline & $\underline{\text { Class } 3}$ & $p_{31}$ & $\underline{p_{32}}$ & $p_{32}$ & $p_{34}$ & $p_{3}$. \\
\hline & $\underline{\text { Class } 4}$ & $\underline{p_{41}}$ & $\underline{p_{42}}$ & $\underline{p_{43}}$ & $\underline{p_{44}}$ & $\underline{p_{4}}$. \\
\hline & $\underline{\text { Total }}$ & $\underline{p \cdot 1}$ & $\underline{p \cdot 2}$ & $\underline{p} \cdot \underline{3}$ & $\underline{p .4}$ & $\underline{1}$ \\
\hline & & & & & & \\
\hline & & & & & & \\
\hline
\end{tabular}




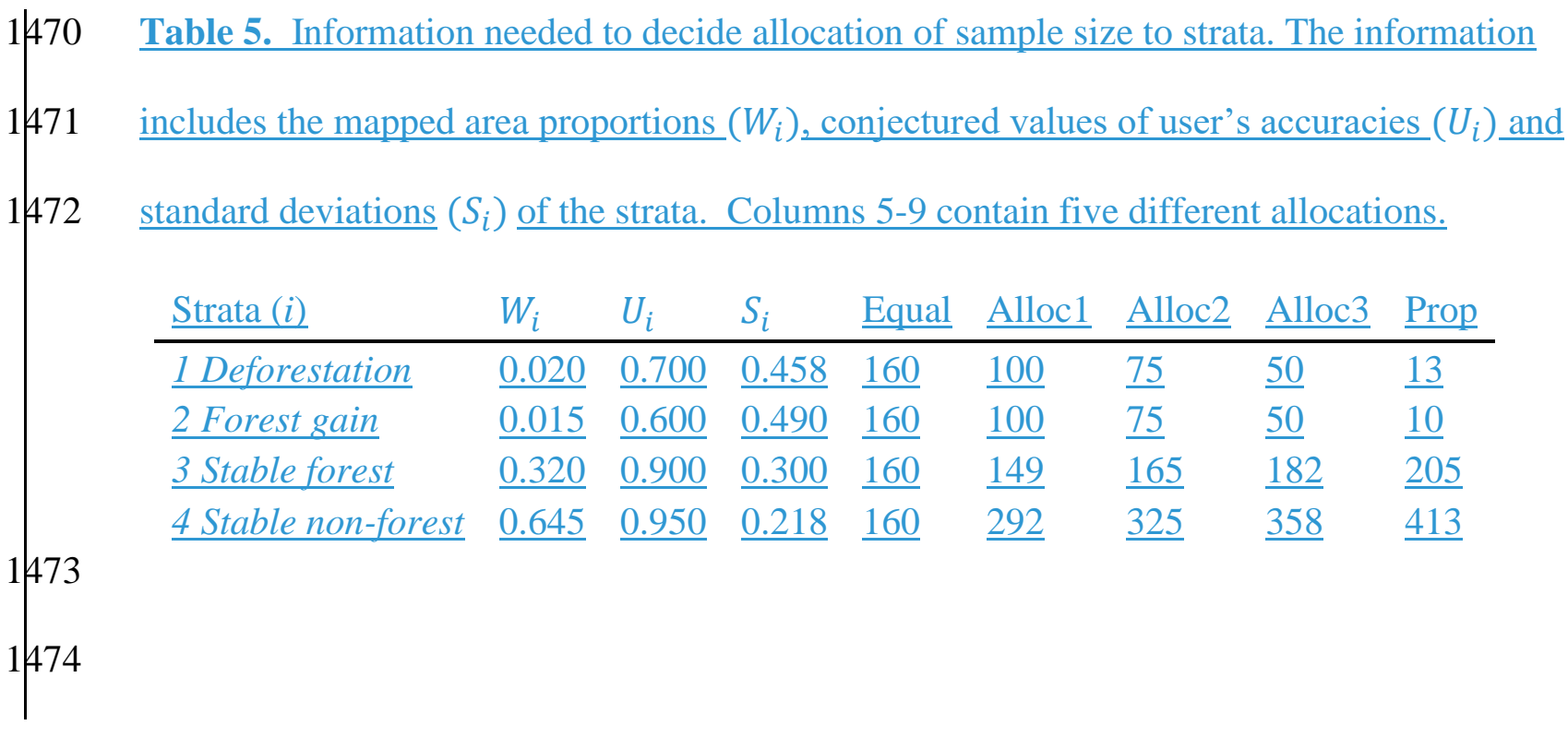


1475 Table 6. Hypothetical population error matrix expressed in terms of proportion of area (see

1476 Section 4) used for sample size and sample allocation planning calculations.

\begin{tabular}{|c|c|c|c|c|c|c|}
\hline \multicolumn{7}{|c|}{$\underline{\text { Reference }}$} \\
\hline- & $\frac{\text { Defore- }}{\text { Station }}$ & $\frac{\text { Forest }}{\text { gain }}$ & $\frac{\text { Stable }}{\text { forest }}$ & $\frac{\text { Stable }}{\text { non-forest }}$ & $\underline{\text { Total }}\left(W_{i}\right)$ & $U_{i}$ \\
\hline Deforestation & $\underline{0.014}$ & $\underline{0}$ & $\underline{0.003}$ & $\underline{0.003}$ & $\underline{0.020}$ & $\underline{0.70}$ \\
\hline Forest gain & $\underline{0}$ & $\underline{0.009}$ & $\underline{0.003}$ & $\underline{0.003}$ & $\underline{0.015}$ & $\underline{0.60}$ \\
\hline Stable forest & $\underline{0.002}$ & $\underline{0}$ & $\underline{0.288}$ & $\underline{0.030}$ & $\underline{0.320}$ & $\underline{0.90}$ \\
\hline Stable non-forest & $\underline{0.004}$ & $\underline{0.002}$ & $\underline{0.025}$ & $\underline{0.614}$ & $\underline{0.645}$ & $\underline{0.95}$ \\
\hline Total & $\underline{0.020}$ & $\underline{0.011}$ & $\underline{0.319}$ & 0.650 & $\underline{1}$ & \\
\hline
\end{tabular}

1477

1478 


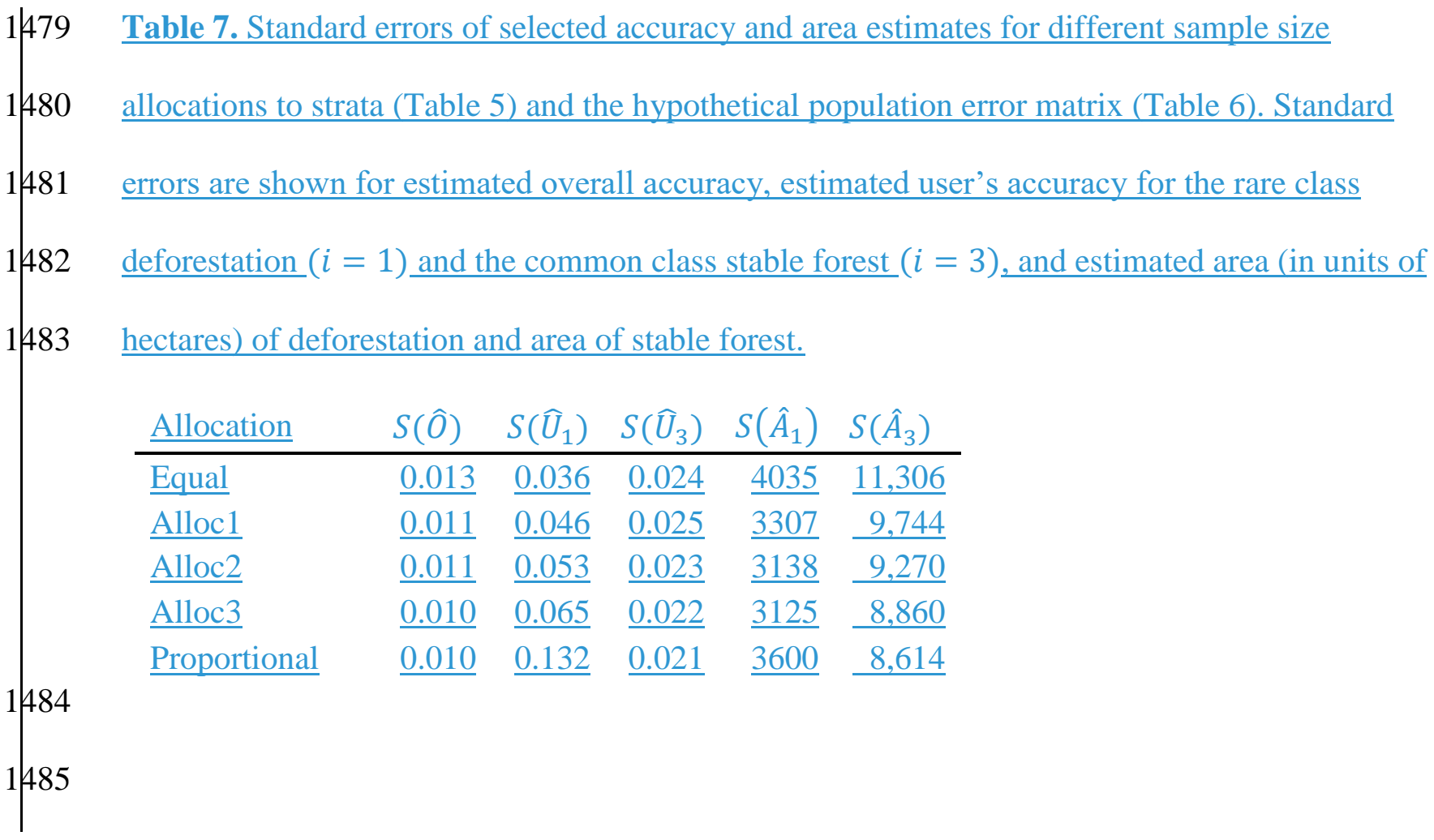




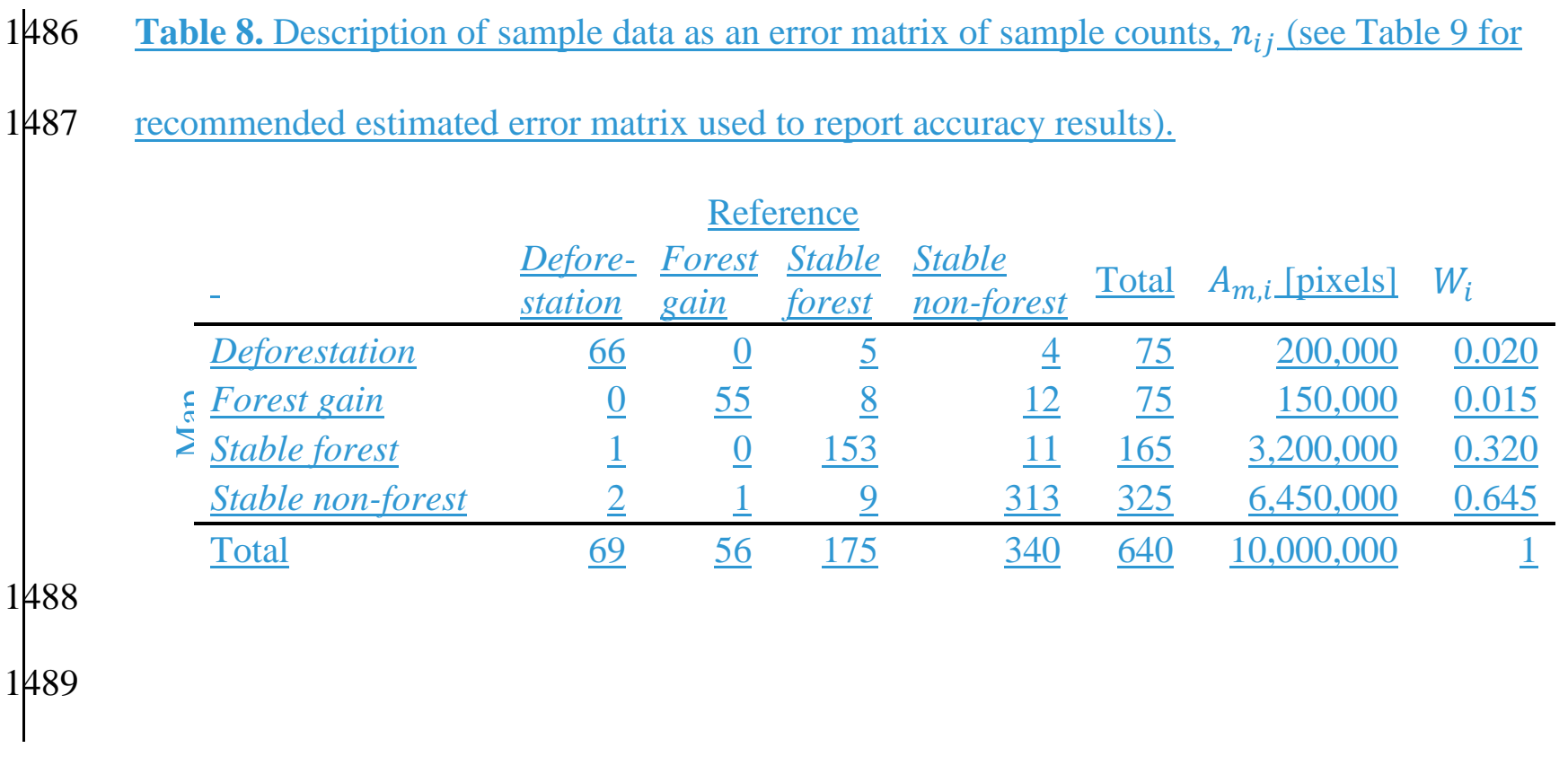




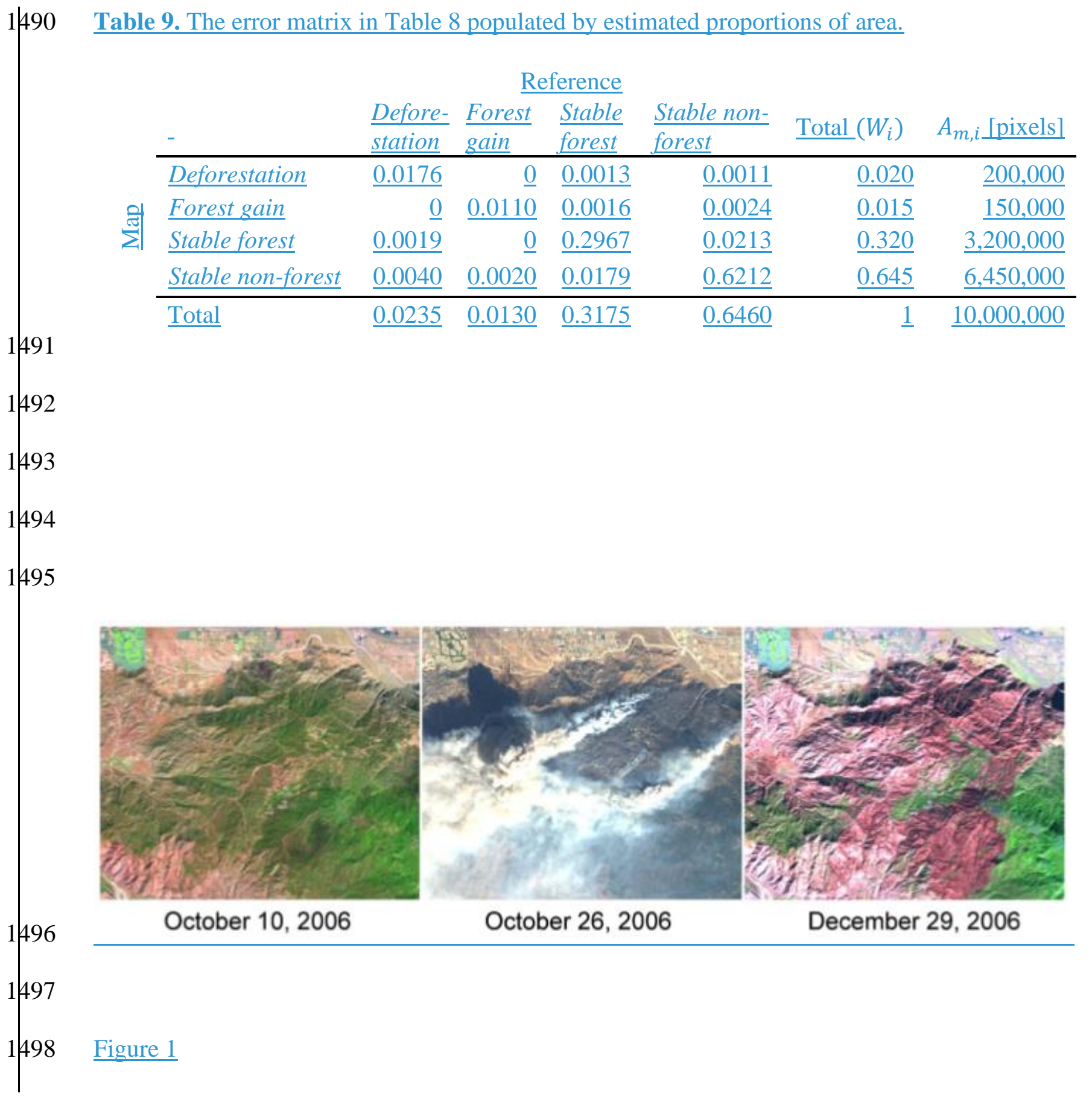

